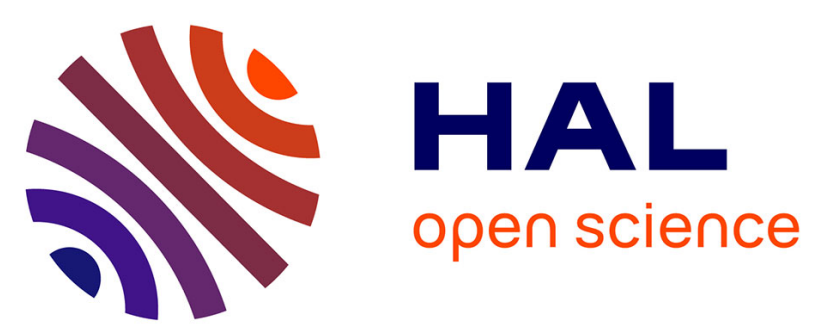

\title{
Theoretical and experimental analysis of co-designed binary phase masks for enhancing the depth of field of panchromatic cameras
}

Alice Fontbonne, Hervé Sauer, François Goudail

\section{- To cite this version:}

Alice Fontbonne, Hervé Sauer, François Goudail. Theoretical and experimental analysis of co-designed binary phase masks for enhancing the depth of field of panchromatic cameras. Optical Engineering, 2021, 60 (03), 10.1117/1.OE.60.3.033101 . hal-03103938v2

\section{HAL Id: hal-03103938 \\ https://hal.science/hal-03103938v2}

Submitted on 1 Mar 2021

HAL is a multi-disciplinary open access archive for the deposit and dissemination of scientific research documents, whether they are published or not. The documents may come from teaching and research institutions in France or abroad, or from public or private research centers.
L'archive ouverte pluridisciplinaire HAL, est destinée au dépôt et à la diffusion de documents scientifiques de niveau recherche, publiés ou non, émanant des établissements d'enseignement et de recherche français ou étrangers, des laboratoires publics ou privés. 


\title{
Theoretical and experimental analysis of co-designed binary phase masks for enhancing the depth of field of panchromatic cameras
}

\author{
Alice Fontbonne ${ }^{*}, 1$, Hervé Sauer $^{1}$, François Goudail ${ }^{1}$ \\ ${ }^{1}$ Université Paris-Saclay, Institut d'Optique Graduate School, CNRS, Laboratoire Charles Fabry, 91127 Palaiseau, \\ France
}

\begin{abstract}
.
We investigate the depth of field (DoF) enhancing capacity of binary annular phase masks embedded in panchromatic imaging systems. We first demonstrate with numerical simulations and real-world imaging experiments that phase masks optimized for monochromatic illumination are somewhat robust to their use under wide spectrum illumination: they provide images that are slightly less sharp but less affected by deconvolution artifacts thanks to spectral averaging. Then, we show that masks specifically optimized for wide spectrum illumination perform better under this type of illumination than monochromatically optimized phase masks under monochromatic illumination, especially when the targeted DoF range is large. This interesting effect comes from the fact that deconvolution artifacts are significantly reduced by wide spectrum illumination. These results show that it is useful to take into account the illumination spectrum together with the scene characteristics and the targeted DoF range for effective co-design of DoF enhancing imaging systems.
\end{abstract}

Keywords: image processing, binary phase masks, deconvolution, optical system codesign, wavefront coding, experimental validation.

*Corresponding author, alice.fontbonne@institutoptique.fr

This pre-print has been published as: Alice Fontbonne, Hervé Sauer, François Goudail, “Theoretical and experimental analysis of co-designed binary phase masks for enhancing the depth of field of panchromatic cameras," Opt. Eng. 60(3), 033101 (2021)

DOI: https://doi.org/10.1117/1.OE.60.3.033101.

\section{Introduction}

Placing a phase mask in the aperture stop of an imaging system can enhance its depth of field (DoF), and this DoF extension capacity can be maximized by jointly optimizing the phase mask and the digital processing algorithm used to deblur the acquired image. This method, introduced by Cathey \& Dowski with a cubic phase mask, ${ }^{1,2}$ has been generalized to different mask models such as continuous rotationally symmetric phase masks, ${ }^{3-5}$ polynomial phase masks, ${ }^{6}$ or different types of binary phase masks. ${ }^{7-11}$ The deblurring algorithm can be based on the Wiener filter ${ }^{12,13}$ or neural networks. ${ }^{14}$ We consider here annular binary phase masks co-optimized with a unique Wiener deconvolution algorithm. Their performance and robustness have been characterized theoretically ${ }^{15}$ and experimentally ${ }^{16}$ in the case of monochromatic illumination. It was shown that nearly optimal performance can be obtained with a limited number of rings and that this performance is robust to small amounts of optical aberrations.

As most binary phase masks are manufactured by binary etching of a dielectric plate, dephasing is wavelength dependent and the optimal behavior is reached for only one wavelength. To mitigate 
this problem, studies have been carried out to use these masks with RGB sensors, optimizing them differently to make them effective in increasing the DoF at three different wavelengths. ${ }^{17-21}$ RGB sensors associated with the co-design of chromatic optics have also been used for various applications (motion deblurring, ${ }^{22}$ depth estimation ${ }^{23,24}$ ). Moreover, several empirical studies on panchromatic imaging applications have shown that the binary phase masks can, in fact, perform correctly in the presence of wide spectrum illumination. ${ }^{25,26}$

The purpose of the present article is to perform a theoretical and experimental study of codesigned DoF enhancing binary phase masks in panchromatic imagers. We first characterize the performance and robustness of phase masks co-optimized for monochromatic imaging when they are used for panchromatic imaging in the visible spectral range. These theoretical analyses are experimentally validated using a real-world imaging experiment. We then quantify the gain in performance brought by taking into account the actual illumination spectrum in the optimization of the masks.

This article is organized as follows: in Section 2, we define our panchromatic imaging model and remind the basics of phase mask optimization. In Section 3, we analyze the behavior of DoF enhancing phase masks in panchromatic imagers in terms of Modulation Transfer Function (MTF) and of final image quality. The results are experimentally validated in Section 4, where we perform imaging experiments with a commercial lens, a CMOS sensor and co-optimized phase masks. In Section 5, we perform co-optimization of phase masks taking into account the actual illumination spectrum. We evaluate the performance gain compared to masks co-optimized for monochromatic light. Section 6 is devoted to concluding remarks and perspectives.

\section{DoF enhancing phase masks in panchromatic imaging systems}

Our goal in this article is to study the behavior of DoF enhancing phase masks in panchromatic imaging systems. In this section, we review the basic concepts necessary for this study. We first define the model for panchromatic MTF, and then how we take into account defocus aberration in it. We finally review the principle of joint optimization of DoF enhancing phase masks and deconvolution algorithms.

\subsection{Wide spectrum illumination}

The main difference between monochromatic and panchromatic systems is in the expression of the Optical Transfert Function (OTF). Considering spatial coordinates $x$ and $y$ in the image plane and spatial frequency coordinates $\nu_{x}$ and $\nu_{y}$, the OTF $\tilde{h}_{\lambda}\left(\nu_{x}, \nu_{y}\right)$ at a chosen wavelength $\lambda$ is given by the Fourier transform of the monochromatic Point Spread Function (PSF) $h_{\lambda}(x, y)$ of the optical system $^{18,27}$ illuminated by monochromatic light at wavelength $\lambda$ :

$$
\tilde{h}_{\lambda}\left(\nu_{x}, \nu_{y}\right)=\iint_{\mathbb{R}^{2}} h_{\lambda}(x, y) \exp \left[-j 2 \pi\left(\nu_{x} x+\nu_{y} y\right)\right] d x d y \text { with } \iint_{\mathbb{R}^{2}} h_{\lambda}(x, y) d x d y=1 .
$$

Let us consider that the scene seen by the imaging system has a mean spectral radiance $R_{\lambda}(\lambda)$ defined over a spectral range $\left[\lambda_{\min }, \lambda_{\max }\right]$. In the following, we assume that the scene is globally neutral and backscatters a mean spectrum proportional to the one of the illuminating source (the sun or an artificial light). Moreover, we consider that the sensor has a spectral sensitivity $S(\lambda)$. 
Hence the polychromatic OTF $\tilde{h}$ has the following expression:

$$
\tilde{h}\left(\nu_{x}, \nu_{y}\right)=\int_{\lambda_{\min }}^{\lambda_{\max }} E(\lambda) \tilde{h}_{\lambda}\left(\nu_{x}, \nu_{y}\right) d \lambda \text { with } E(\lambda)=\frac{R_{\lambda}(\lambda) \times S(\lambda)}{\int_{\lambda_{\min }}^{\lambda_{\max }} R_{\lambda}(\lambda) \times S(\lambda) d \lambda} .
$$

where $E(\lambda)$ denotes the normalized "effective" imaging spectrum. In practice, for numerical simulation, this integration over the wavelengths is approximated by discretizing the effective spectrum (as it is done in optical design software packages like Synopsys $\mathrm{CodeV}^{\circledR}$ or Zemax Optic Studio ${ }^{\circledR}$ ). We noticed that, in the visible range, a discretization with wavelengths separated by $40 \mathrm{~nm}$ is sufficient. We denote $\Lambda$ such a discretized set of wavelengths in $\left[\lambda_{\min }, \lambda_{\max }\right]$. Hence the OTF obtained on the sensor after the optical system is modeled by:

$$
\tilde{h}_{\Lambda}\left(\nu_{x}, \nu_{y}\right)=\sum_{\lambda \in \Lambda} E(\lambda) \tilde{h}_{\lambda}\left(\nu_{x}, \nu_{y}\right) \text { with } \sum_{\lambda \in \Lambda} E(\lambda)=1 .
$$

\subsection{Defocus aberration}

The shape of the PSF or OTF not only depends on the spectrum chosen for illumination, but also on the aberrations of the optical system. The most important one to consider when trying to increase the DoF is of course the defocus aberration induced by the object distance range. It is convenient to parameterize the defocus with the signed defocus wavefront error at the edge of the exit pupil, $\psi$, that is, the optical path difference on the marginal rays between the actual defocused wavefront and the reference sphere. Assuming that the exit pupil is far from the image plane, and using a first non zero order expansion in the image Numerical Aperture $N A$ and in the image distance shift $\Delta z_{i}$, this parameter can be written, at a given wavelength $\lambda_{0}$, as:

$$
\psi\left(\lambda, \Delta z_{o}\right) \approx \frac{1}{2}\left(z_{i 0} N A\right)^{2}\left(\frac{1}{z_{i 0}}+\frac{1}{z_{o 0}+\Delta z_{o}}-\frac{1}{f^{\prime}(\lambda)}\right)
$$

where $f^{\prime}(\lambda)$ is the effective focal length, $z_{o 0}$ is the distance between the nominal object position and the lens object principal plane $H$ (taken as positive for real objects in front of the lens) and $z_{i 0}$ is the distance between the lens image principal plane $H^{\prime}$ and the nominal image plane, which is also the fixed detector position. According to the Rayleigh criterion, the defocus parameter range in which the image quality can be considered nearly as good as if it were focused is $\left[\psi_{\min }, \psi_{\max }\right]=$ $\left[-\frac{\lambda_{0}}{4}, \frac{\lambda_{0}}{4}\right]$.

Note that when implementing a real DoF enhancing system in Section 4, we will check that the axial chromatic aberration of the used commercial achromatic imaging lens $\Delta f^{\prime}(\lambda)$ is negligible,

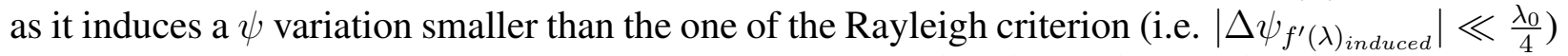
for the used numerical aperture. Therefore, considering $\psi\left(\Delta z_{o}\right)=\psi\left(\lambda_{0}, \Delta z_{o}\right)$ is sufficient in our case.

\subsection{Co-designed phase masks for DoF-extended monochromatic imaging}

We extend the DoF of an imaging system by placing a binary annular phase mask in its aperture stop. These masks are static spatial phase modulating optical elements consisting of a series of $N$ concentric annular regions of phase modulation of alternatively 0 or $\pi$ radians at the nominal wavelength $\lambda_{0}$ (Fig. 1). Each annular constant-phase area corresponds to a so-called ring, so that 
a $N$-ring mask of clear aperture radius $R$ is parameterized by $N-1$ normalized radius values $\phi=\left\{\rho_{1}, \ldots, \rho_{N-1}\right\}$, where the radius of the $i$ 'th phase transition is $r_{i}=\rho_{i} R$ and $\phi$ satisfies the conditions $0<\rho_{1}<\ldots<\rho_{N-1}<\rho_{N}=1$.

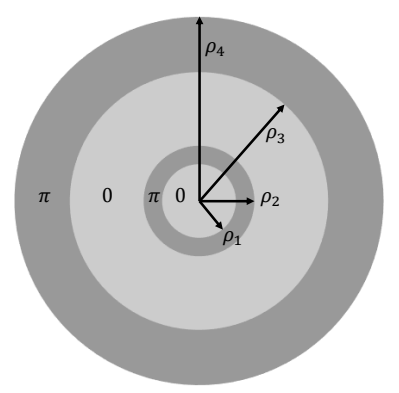

Fig 1 Example of a binary phase mask consisting of a series of 4 concentric annular regions of phase modulation of alternatively 0 (light gray) or $\pi$ radians (dark gray) at the nominal wavelength $\lambda_{0}$.

In practice, the binary phase masks we use are manufactured as binary depth modulation on fused silica substrates using UV photolithography associated with Ion Beam Etching (IBE) followed by Inductive Coupled Plasma (RIE - ICP) etching. The etching depth $e$ is calculated so that the phase shift between two rings is equal to $\pi$ radians at the chosen nominal wavelength $\lambda_{0}$, according to the formula:

$$
e=\frac{\lambda_{0}}{2\left(n_{\lambda_{0}}-1\right)}
$$

where $n_{\lambda_{0}}=1.4601$ is the refractive index of fused silica at $\lambda_{0}=545 \mathrm{~nm}$. This leads to a required etching depth of $e=592 \mathrm{~nm}$.

Under monochromatic illumination at wavelength $\lambda_{0}$, the image produced on the sensor when the phase mask is placed in the pupil can be modeled by $h_{\lambda_{0}, \psi}^{\phi}(r) * O(r)$, where $O(r)$ is the ideal sampled scene image ( $r$ represents the spatial coordinates), $*$ denotes the convolution operator, and $h_{\lambda_{0}, \psi}^{\phi}(r)$ is the PSF of the optical system containing a phase mask characterized by $\phi$ for a defocus parameter of $\psi$. The image is then deconvolved with a digital deconvolution filter $w_{\lambda_{0}}^{\phi}(r)$ to restore its sharpness. The restored image at the output of this hybrid optical-digital system can be modeled as:

$$
\hat{O}(r)=w_{\lambda_{0}}^{\phi}(r) *\left[h_{\lambda_{0}, \psi}^{\phi}(r) * O(r)+n(r)\right]
$$

where $n(r)$ is the detection noise. The mean-squared error (MSE) of the restored image is then defined as:

$$
M S E(\phi, \psi)=\mathrm{E}\left[\int|\hat{O}(r)-O(r)|^{2} d r\right]
$$

where $E[\cdot]$ represents the mathematical expectation over the noise $n(r)$ and the scene image $O(r)$, which are both assumed to be zero-mean, stationary stochastic processes of power spectral density (PSD) $S_{n n}(\nu)$ and $S_{o o}(\nu)$ respectively, with $\nu$ representing the spatial frequency coordinates. We model the object PSD $S_{o o}(\nu)$ by the function $\propto \nu^{-2.5}$, which is a realistic model of natural scenes. ${ }^{15,16,28,29}$ In addition, we will consider in the remainder of the paper that, unless otherwise 
stated, the PSD of the noise $S_{n n}(\nu)$ is white and adjusted so that the signal-to-noise ratio (SNR) is $34 \mathrm{~dB}$. The goal of co-design is to find the deconvolution filter $w_{\lambda_{0}}^{\phi}(r)$ and the mask parameters $\phi$ that minimize $\operatorname{MSE}(\phi, \psi)$ over the targeted range of defocus parameters. This range is discretized over $K$ defocus parameter values so that $\psi \in\left\{\psi_{1}, \psi_{2}, \cdots, \psi_{K}\right\}$. For deconvolution, we use the averaged Wiener filter that has been proved to be the optimal one ${ }^{12}$ and has the following expression in the Fourier space:

$$
w_{\lambda_{0}}^{\phi}(\nu)=\frac{\frac{1}{K} \sum_{k=1}^{K}\left[\widetilde{h}_{\lambda_{0}, \psi_{k}}^{\phi}(\nu)\right]^{\star}}{\frac{1}{K} \sum_{k=1}^{K}\left|\widetilde{h}_{\lambda_{0}, \psi_{k}}^{\phi}(\nu)\right|^{2}+\frac{S_{n n}(\nu)}{S_{o o}(\nu)}}
$$

where the superscript $\star$ denotes the complex conjugate. The parameters $\phi_{\text {opt }}$ of the optimal mask are optimized using a "minimax" criterion:

$$
\phi_{\text {opt }}=\arg \min _{\phi}\left\{\max _{k}\left[\operatorname{MSE}\left(\phi, \psi_{k}\right)\right]\right\}
$$

We have used this optimization method to design two binary phase masks. The first one, denoted " $1 \lambda_{0}$-mask", is the optimal mask ${ }^{15}$ for a targeted defocus parameter range of $\left[-1 \lambda_{0}, 1 \lambda_{0}\right]$, which is 4 times the natural DoF of the lens. It has three rings. The second one, denoted " $2 \lambda_{0}$-mask", is optimal for a targeted defocus parameter range of $\left[-2 \lambda_{0},-2 \lambda_{0}\right]$, which corresponds to extend 8 times the natural DoF of the lens. It has six rings. Their normalized outer radii are given in Table 1 and their profiles are represented on Fig. 2. Considering a phase mask manufactured with a total radius equal to $2.25 \mathrm{~mm}$, we notice that the minimal distance between two phase steps is $130 \mu \mathrm{m}$. The positioning uncertainty of the rings in our manufacturing process is better than one micrometer, so we are confident to manufacture these optimal masks with a sufficient accuracy for the relevancy of our experimental tests.

\begin{tabular}{|c|c|c|c|c|c|c|}
\hline Phase mask & $\mathrm{e}$ & $\rho_{1}$ & $\rho_{2}$ & $\rho_{3}$ & $\rho_{4}$ & $\rho_{5}$ \\
\hline $1 \lambda_{0}$-mask & 592 & 0.7684 & 0.9272 & & & \\
$1 \lambda_{0}$-polychromatic mask & 722 & 0.2412 & 0.8416 & & & \\
$2 \lambda_{0}$-mask & 592 & 0.1307 & 0.6533 & 0.7917 & 0.9192 & 0.9771 \\
$2 \lambda_{0}$-polychromatic mask & 647 & 0.0992 & 0.6391 & 0.8397 & 0.9410 & \\
\hline
\end{tabular}

Table 1 Normalized radii of the optimal phase masks obtained under monochromatic spectrum assumption $\left(1 \lambda_{0^{-}}\right.$ mask and $2 \lambda_{0}$-mask) and under wide spectrum assumption ( $1 \lambda_{0}$-polychromatic mask and $2 \lambda_{0}$-polychromatic mask are defined in Section 5). The optimal step height $e$ is given in nanometers.

These masks have been optimized for monochromatic imaging at wavelength $\lambda_{0}$. Our goal in the following is to evaluate their behavior under wide spectrum illumination by simulation (Section 3) and experimentation (Section 4). In Section 5, we will compare by simulation their performance with those of masks optimized for wide spectrum illumination.

\section{Performance of DoF-enhancing phase masks in panchromatic imaging}

Since most binary phase masks (in particular the ones we use) are built with simple binary etching in a dielectric substrate that gives $\pi$ radians-phase steps at $\lambda_{0}$, their behavior changes when they are used at another wavelength. Some approaches have been explored in order to mitigate this problem. For instance, it is possible to use a superposition of two binary phase masks, one "positive" and 
(a)

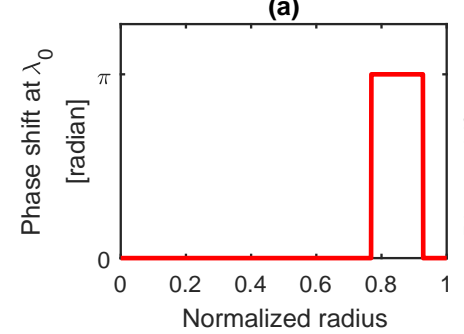

(c)

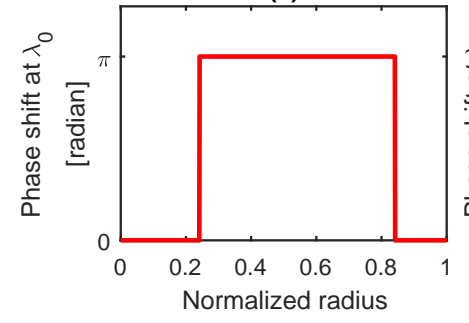

(b)

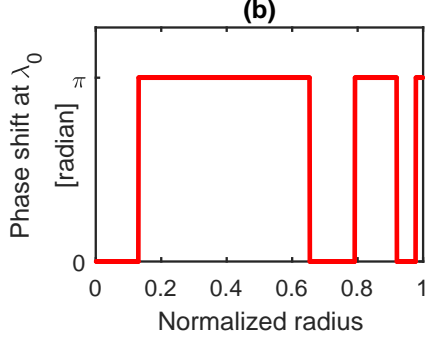

(d)

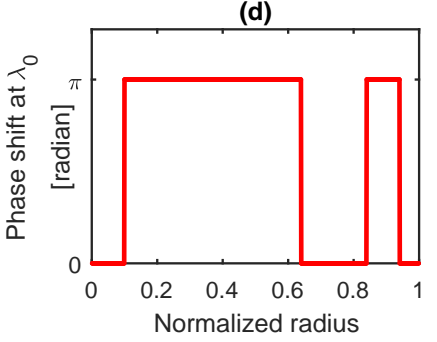

Fig 2 Phase shift profile of the phase masks at the nominal wavelength $\lambda_{0}$. (a) $1 \lambda_{0}$-mask (b) $2 \lambda_{0}$-mask (c) $1 \lambda_{0^{-}}$ polychromatic mask (d) $2 \lambda_{0}$-polychromatic mask.

one "negative", to force the phase shift to be close to $\pi$ for three chosen RGB wavelengths thanks to the choice of the dispersion of two different glasses. ${ }^{20}$ Another method, in case of a color sensor, can be on the contrary to make the response of a chromatic element different for three wavelengths and to select the high frequency content from the sharpest channel. ${ }^{19,23}$

Our purpose in this section is to study in detail the behavior of masks optimized for a given wavelength when used with another one or with a wide spectrum, and to evaluate the robustness of DoF enhancement ability to wavelength discrepancy. We first study the variations of the MTF with the imaging spectrum, then the performance of the whole digital-optical DoF-enhancing system.

\subsection{Polychromatic PSFs and MTFs}

The ability of a phase mask to improve DoF can be easily grasped from the behavior of its MTF as the defocus parameter $\psi$ varies. We represent in Fig. 3(a) the MTFs of the $1 \lambda_{0}$-mask for different values of the defocus parameter up to $\psi=1.5 \lambda_{0}$ when imaging at the reference wavelength $\lambda_{0}$. We can see that the MTFs are quite close to each other within the targeted $\psi$ range $\left[-1 \lambda_{0}, 1 \lambda_{0}\right]$ but collapse outside this range (for example, at $\psi=1.5 \lambda_{0}$ ). Moreover, we can notice that, in a perfect optical system with only defocus aberration, the MTFs corresponding to opposite defocus $\psi$ and $-\psi$ are exactly superposed. This is due to the fact that the phase shift between successive rings of the mask is $\pi$ radians at the monochromatic wavelength $\lambda_{0}$, and that $+\pi$ or $-\pi$ radians are equivalent phases modulo $2 \pi$. Therefore, the $1 \lambda_{0}$-mask has exactly the same behavior on the $\left[-1 \lambda_{0}, 0\right]$ and the $\left[0,1 \lambda_{0}\right]$ defocus ranges. This allows to reduce the computation required for optimization in the whole defocus parameter range $\left[-\psi_{\max }, \psi_{\max }\right]$.

Let us now study the MTFs of this mask at monochromatic illumination with two other wavelengths: $\lambda_{B}=450 \mathrm{~nm}$ (Fig. 3(b)) and $\lambda_{R}=650 \mathrm{~nm}$ (Fig. 3(c)). Note that when the illumination wavelength is different from the nominal one, the phase shift between the rings of the phase mask differs from $\pi$ radians: it is multiplied by a factor $\frac{\lambda_{0}}{\lambda} \times \frac{n_{\lambda}-1}{n_{\lambda_{0}}-1}$, where $\lambda$ is the real illumination 
(a)

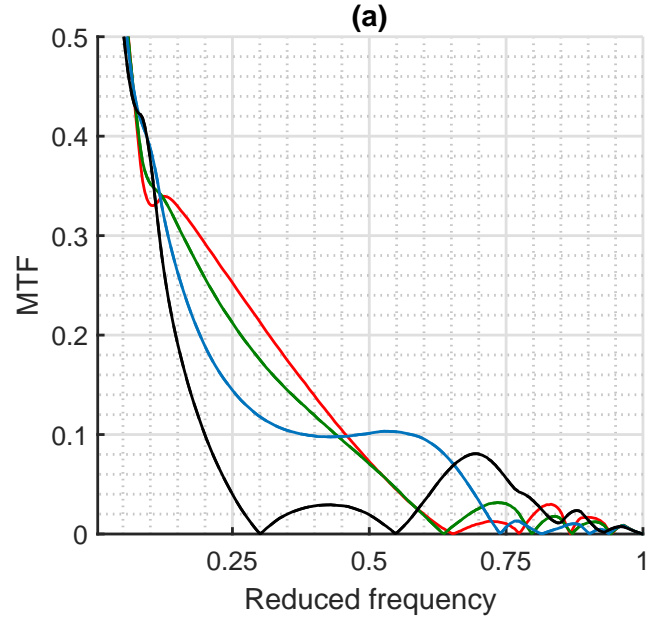

(c)

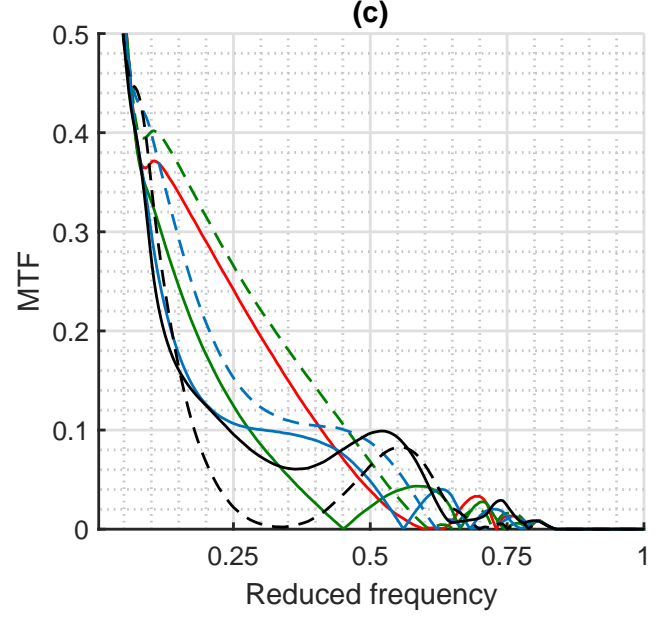

(b)

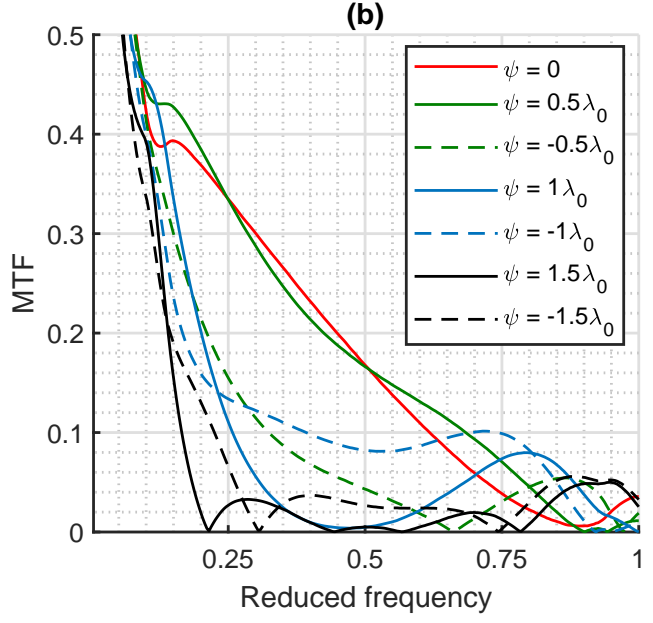

(d)

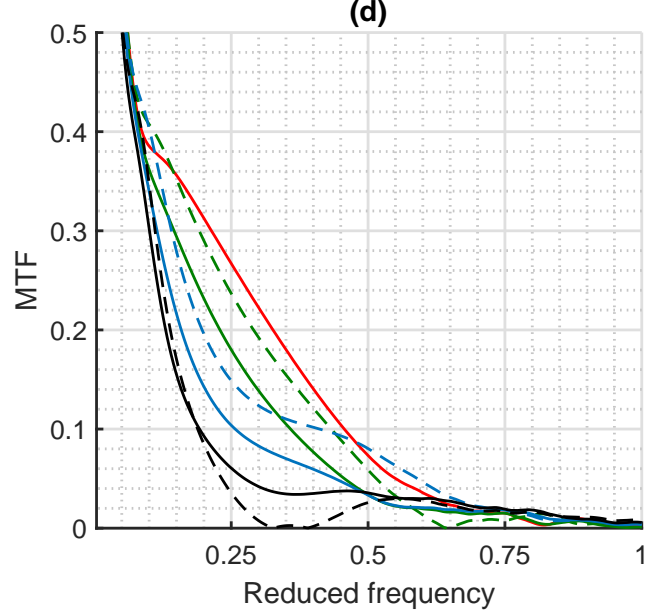

Fig 3 MTFs for several defocus for the $1 \lambda_{0}$-mask integrated in a perfect lens imaging a scene illuminated with different spectrum. (a) monochromatic illumination at $\lambda_{0}$ (b) monochromatic illumination at $\lambda_{B}=450 \mathrm{~nm}$ (c) monochromatic illumination at $\lambda_{R}=650 \mathrm{~nm}$ (d) wide spectrum illummination of the sun light. The $\mathrm{x}$-axis is expressed with reduced frequencies $\frac{f}{f_{0}}$, calculated from the cutoff spatial frequency at $\lambda_{0}$ and with a F-number $F \#, f_{0}=\frac{1}{\lambda_{0} F \#}$. For all graphs, the $\psi$-legend is the same as in (b).

wavelength, and $n_{\lambda}$ is the index of the material in which the mask is engraved at $\lambda$. In our case, this material is fused silica. We can neglect the coefficient $\frac{n_{\lambda}-1}{n_{\lambda_{0}}-1}$ because, for all the wavelengths in the visible spectrum, the difference it brings to the phase shift is small $(<4 \%)$ and less than the difference brought by a defect on the step height $e$ of $\pm 20 \mathrm{~nm}$, which is within the tolerances of the manufacturing process.

Let us first consider Figure 3(b), where the behavior of the $1 \lambda_{0}$-mask at $\lambda_{B}$ is plotted. We notice that since the illumination wavelength is different from the nominal one, the mask behavior is no longer symmetric with respect to the sign of the defocus parameter $\psi$. Moreover, the imaging 
system natural cutoff frequency at this wavelength is $f_{B}=\frac{1}{\lambda_{B} F \#}$, where $F \#$ denotes the F-number of the lens. It is larger than the cutoff frequency at $\lambda_{0}, f_{0}=\frac{1}{\lambda_{0} F \#}$, that corresponds to the reduced frequency equal to 1 in the graphs of Fig. 3. This is why in Fig. 3(b), the MTFs do not reach 0 at the reduced frequency 1 . Another similar consequence is that the MTF at the focus position $\psi=0$ is raised, as would be the diffraction limited MTF drawn with the same x-axis scale. For example, the MTF is equal to 0.26 at the reduced frequency 0.25 with an illumination wavelength $\lambda_{0}$ (Fig. 3(a)) but is equal 0.34 at the same reduced frequency with an illumination wavelength $\lambda_{B}$. We notice that the MTF is raised not only on focus but also, for instance, at $\psi=0.5 \lambda_{0}$. However, all the other curves collapse, and in particular, the one for $\psi=+1 \lambda_{0}$ : the $1 \lambda_{0}$-mask cannot extend the DoF up to $\psi=+1 \lambda_{0}$ anymore at $\lambda_{B}$. Futhermore, the MTF curves spanning the targeted DoF range are now so different that it would be difficult for a unique deconvolution filter to perform correctly.

Let us now consider illumination at $\lambda_{R}$. We observe in Fig. 3(c) that the MTFs reach zero at the reduced frequency 0.85 , because the cutoff frequency of the imaging system at $\lambda_{R}$ is smaller than $f_{0}$. This has the effect of grouping together the MTFs obtained for the different defocus values, and in this particular case, the MTF at defocus $\psi=1.5 \lambda_{0}$, which is outside the targeted defocus range, is larger. However, this improvement in terms of grouping is not without a significant decline in the overall level of the MTFs. At the reduced frequency 0.5, the MTF values which were larger than 0.08 are now all smaller. Therefore, the $1 \lambda_{0}$-mask could be used with a monochromatic wavelength $\lambda_{R}$ and improve the DoF as well (or better) than the targeted one, only if the SNR is sufficient to ensure correct deconvolution and if the scene PSD $S_{o o}(\nu)$ is not too broad.

With these curves, we can therefore see that binary phase masks are not good candidates for use in imaging with a narrow spectrum different from that for which they have been optimized. Let us now consider wide spectrum imaging with $R_{\lambda}(\lambda)$ equal to the sun spectrum (a simple model of a black body at $5778 \mathrm{~K}$ is taken) and $S(\lambda)$ to the sensitivity of a silicon sensor, given a sun effective spectrum $E(\lambda)$ presented Fig. 4. Using Eq. (3), we obtain the MTF curves in Fig. 3(d). We notice that the weighted average of the different MTFs at $\psi=0$ is close to the MTF obtained with a monochromatic illumination at $\lambda_{0}$. For example, the polychromatic MTF at the reduced frequency 0.35 is equal to 0.18 , as for the monochromatic MTF at $\lambda_{0}$, whereas it was equal to 0.27 at $\lambda_{B}$ and 0.15 at $\lambda_{R}$. Generally speaking, for all defocus positions, the MTFs are smoothed out by spectral averaging (see Eq. (3)). Therefore, we can observe that the polychromatic MTFs are closer to each other than the MTFs at $\lambda_{B}$ and higher than those at $\lambda_{R}$.

We have represented in Fig. 5 the same set of curves as in Fig. 3 for the $2 \lambda_{0}$-mask. The conclusions are very similar : for monochromatic illumination at $\lambda_{0}$, the optimized $2 \lambda_{0}$-mask can extend the DoF to a $\psi$ range of $\left[-2 \lambda_{0}, 2 \lambda_{0}\right]$ because the MTF curves are close together (Fig. 5(a)). When the mask is used with a $\lambda_{B}$ illumination, the best MTF (at the focus position $\psi=0$ ) is higher, but the MTFs are distant from each other over the defocus range (Fig. 5(b)). When the mask is used with a $\lambda_{R}$ illumination, all the MTFs are more grouped together but at a very low level (Fig. 5(c)). The $2 \lambda_{0}$-mask is thus not efficient at these wavelengths but still has an interesting behavior under wide spectrum illumination (Fig. 5(d)) since in this case, the MTFs are weighted averages of the MTFs at the different wavelengths making up the spectrum. It is particularly noticeable that the polychromatic MTFs are less "bumpy" that the monochromatic ones.

One of the main characteristics of the panchromatic MTFs is to be smooth, for both $1 \lambda_{0}$-mask and $2 \lambda_{0}$-mask. In order to analyze how this characteristic is translated in the direct space, we have 


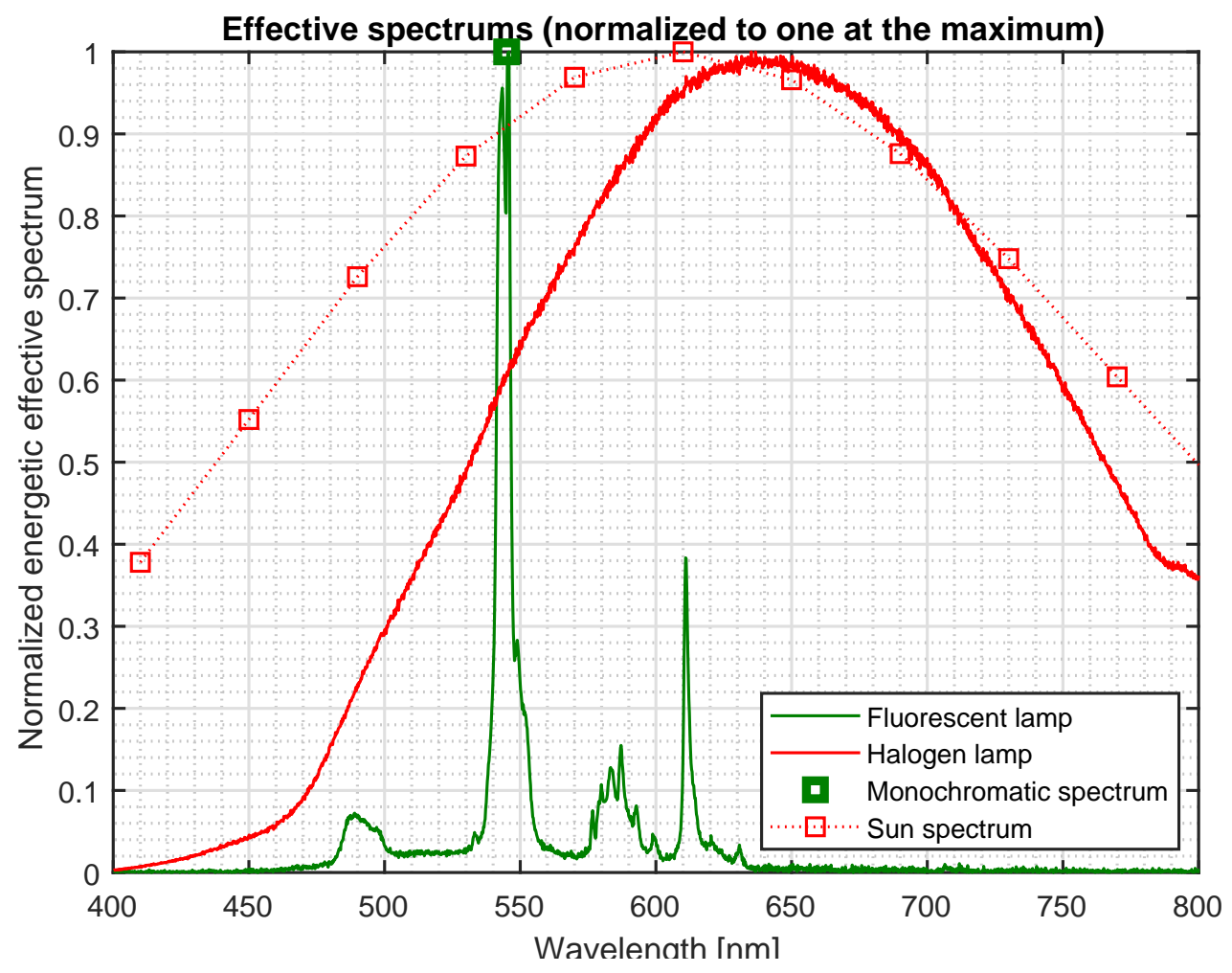

Fig 4 Sun effective spectrum and monochromatic effective spectrum at $\lambda_{0}$ used during theoretical validations (Section 3 and 5) and effective spectrum of a fluorescent lamp with green filter and an halogen lamp based on their mesured spectrums used during experimentation (Section 4). For the sake of clarity, all the spectrums are represented normalized to one at the maximum.

plotted in Fig. 6 the profile of the PSF as a function of the defocus parameter $\psi$ in the presence of monochromatic illumination at $\lambda_{0}$ and in the presence of wide spectrum illumination. Since the PSF of a perfect lens with an annular binary phase mask is rotationally symmetric, a crosssection through the center of the PSF is sufficient to fully represent it. We have thus represented this slice along the vertical axis, the colorbar giving the local image plane irradiance with Strehl normalization, i.e. the irradiance divided by the maximum irradiance given by a perfect lens of the same numerical aperture. This PSF slice is plotted for all values of the defocus parameter $\psi$ ranging from -1.5 to 1.5 times the $\psi_{\max }$ value of the DoF range $\left[-\psi_{\max }, \psi_{\max }\right]$ for which the phase masks have been optimized. On these graphs, it is observed that the PSF of the $1 \lambda_{0}$-mask under wide spectrum illumination (Fig. 6(b)) is slightly less symmetrical along the $\psi$ axis than under monochromatic illumination at $\lambda_{0}$ (Fig. 6(a)). For this small DoF range, the obtained PSFs stay however similar to each other along the defocus range and have few rings. Similarly, we observe that the $2 \lambda_{0}$-mask used with wide spectrum illumination (Fig. 6(d)) is less symmetric over the defocus parameter range than with monochromatic illumination at $\lambda_{0}$ (Fig. 6(c)).

In the next section, we will see how MTF (or PSF) variations along the defocus range, that are mostly present under monochromatic illumination, can induce deconvolution artifacts that are detrimental to DoF enhancement performance. 
(a)

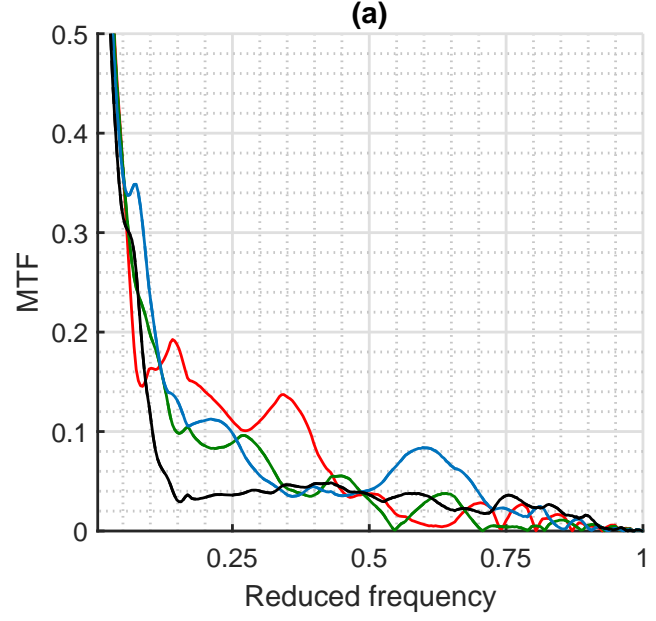

(c)

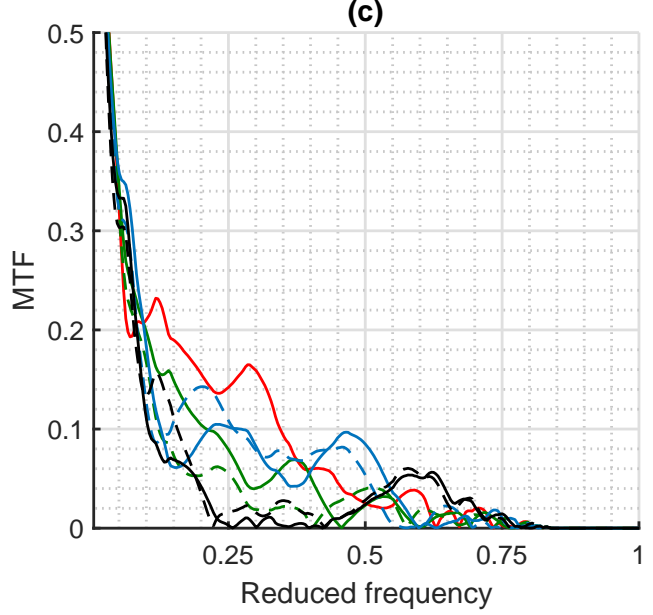

(b)

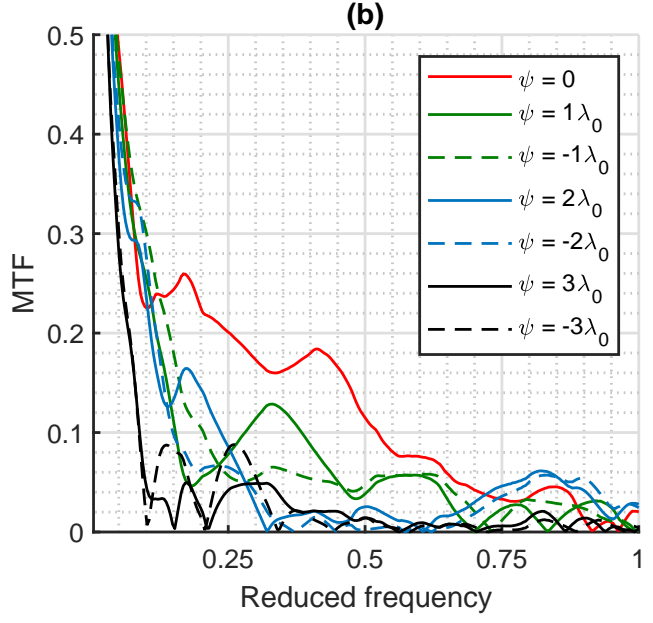

(d)

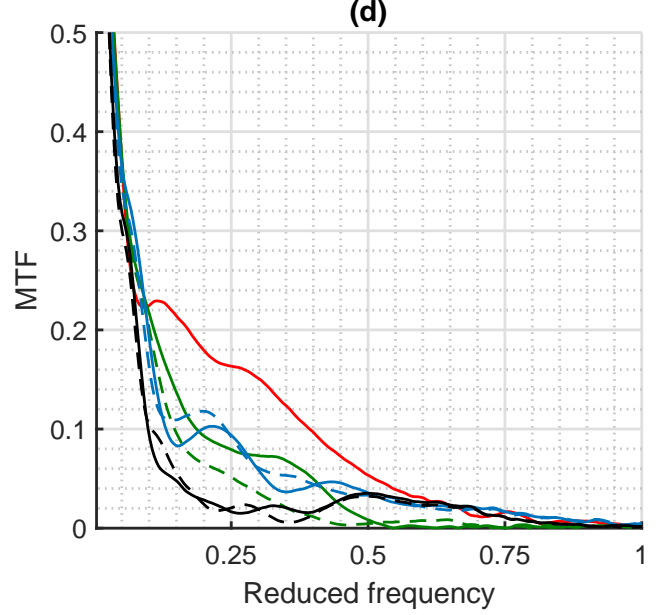

Fig 5 MTFs for several defocus for the $2 \lambda_{0}$-mask integrated in a perfect lens imaging a scene illuminated with different spectrum. (a) monochromatic illumination at $\lambda_{0}$ (b) monochromatic illumination at $\lambda_{B}=450 \mathrm{~nm}$ (c) monochromatic illumination at $\lambda_{R}=650 \mathrm{~nm}$ (d) wide spectrum illummination of the sun. The $\mathrm{x}$-axis is expressed with reduced frequencies, calculated from the cutoff spatial frequency at $\lambda_{0}, f_{0}=\frac{1}{\lambda_{0} F \#}$. For all graphs, the $\psi$-legend is the same as in (b).

\subsection{DoF enhancement performance in polychromatic imaging}

Let us now analyze the influence of illumination on the end-to-end behavior of the imaging system including the deconvolution process. Figure 7(a) represents the image obtained with an on focus $(\psi=0)$ imaging system without phase mask at $\lambda_{0}$ and Fig. 7(d) the image when the system is defocused of $\psi=1 \lambda_{0}$. The blurring due to defocus is obvious. Figures 7(b) and 7(e) represent the restored image obtained for the two same values of $\psi$ when the system is equipped with the $1 \lambda_{0}$-mask and illuminated by monochromatic light at wavelength $\lambda_{0}$. The averaged Wiener filter defined in Eq. (8) has been used for deconvolution. Figures 7(c) and 7(f) represent the same im- 
(a)

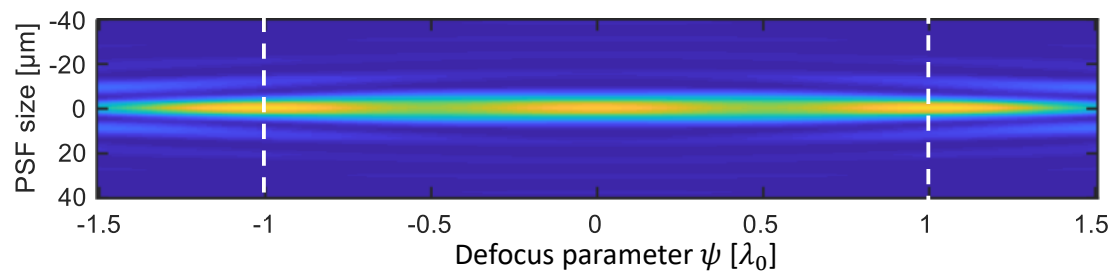

(b)

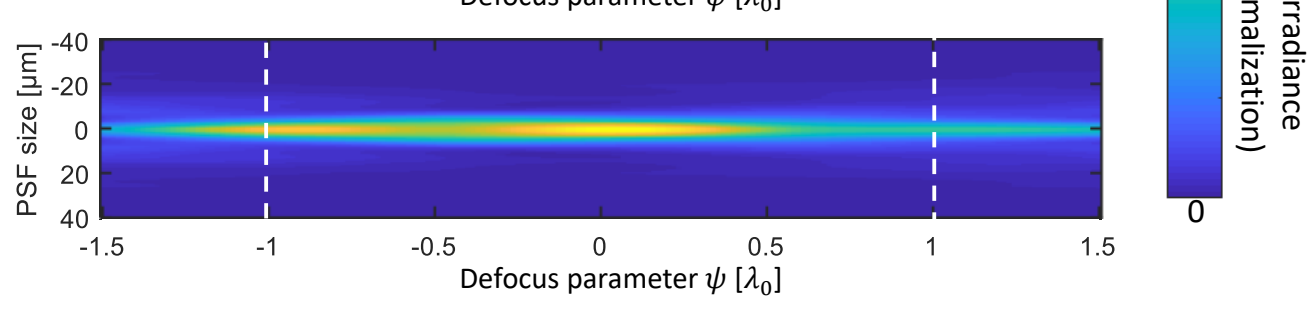

(c)

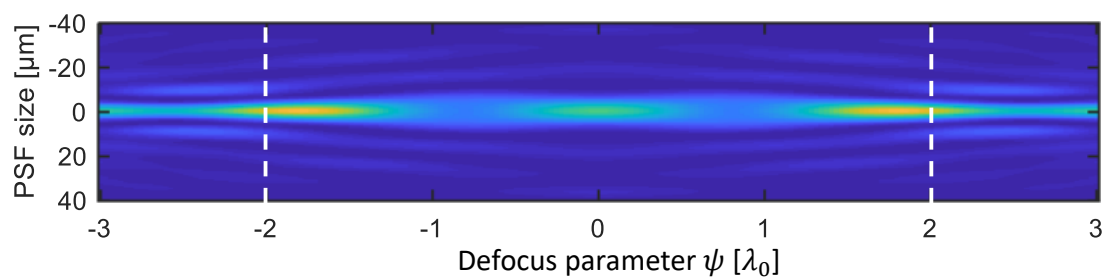

(d)
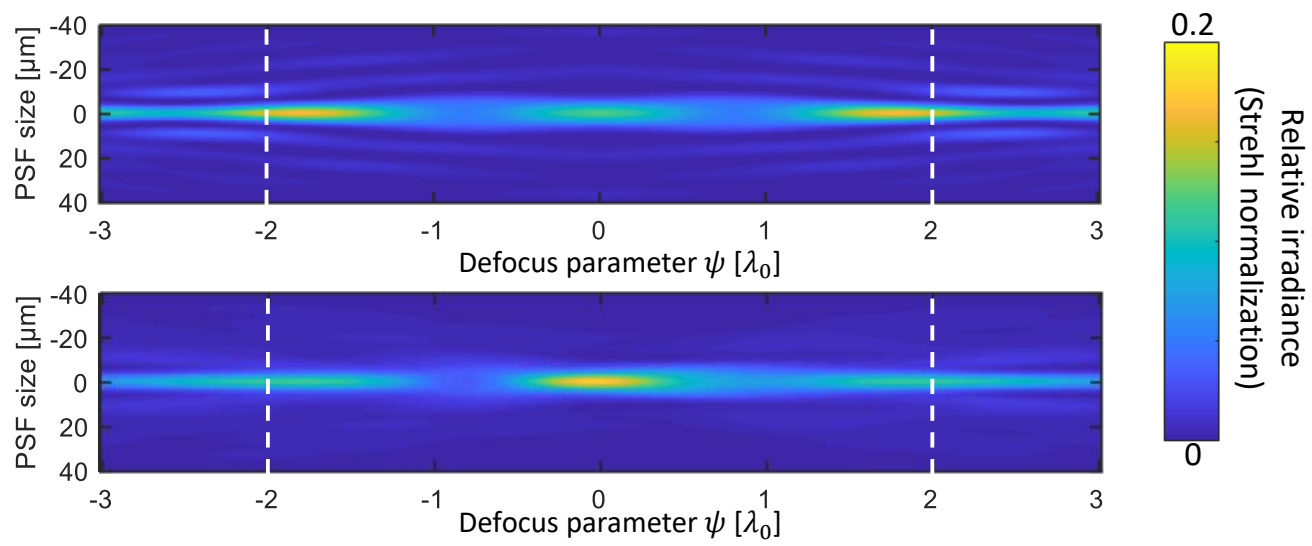

Fig 6 Slice of the PSF versus the defocus parameter obtained for different illumination of a perfect lens associated with a phase mask. (a) The $1 \lambda_{0}$-mask at the monochromatic wavelength $\lambda_{0}$, (b) the $1 \lambda_{0}$-mask with a wide spectrum, (c) the $2 \lambda_{0}$-mask at the monochromatic wavelength $\lambda_{0}$ and (d) the $2 \lambda_{0}$-mask with a wide spectrum. For all graphs, the limits of the DoF for which the masks have been optimized are marked by white dashes.

ages when illumination is polychromatic with the spectrum of the sun. Deconvolution has been performed with the average Wiener filter built with the $\tilde{h}_{\lambda_{0}, \psi}^{\phi}$ in Eq. (8) replaced by the polychromatic $\tilde{h}_{\Lambda, \psi}^{\phi}$ as given in Eq. (3).

We can see that the restored images under wide spectrum illumination have a good sharpness, although the mask was not optimized for this illumination. This shows the robustness of the mask to such kinds of changes in operational conditions. In particular, the images on focus $(\psi=0)$ in Fig. 7(b) and (c) are nearly as sharp as the image obtained without a phase mask (Fig. 7(a)). However, for a defocus parameter $\psi=1 \lambda_{0}$, the image under wide spectrum illumination (Fig. 7(f)) is slightly more "blurry" than the one under monochromatic illumination at $\lambda_{0}$ (Fig. 7(e)). Thus, as predicted from the MTF shapes in Fig. 6(a) and (b), the $1 \lambda_{0}$-mask performs slightly better under monochromatic illumination at $\lambda_{0}$ than under wide spectrum illumination. In order to quantify this difference, we have measured the minimal quality of the restored images over the whole targeted 
DoF range with the following "image quality" (IQ) criterion defined from Eq. (7) as:

$$
I Q_{\text {min }}=\min _{k}\left[10 \log _{10} \frac{1}{\operatorname{MSE}\left(\phi, \psi_{k}\right)}\right]=10 \log _{10} \frac{1}{\max _{k} \operatorname{MSE}\left(\phi, \psi_{k}\right)} .
$$

To obtains the values given in Tab. 2, we have assumed that the object PSD $S_{o o}(\nu)$ is proportional to $\nu^{-2.5}$ and the SNR is $34 \mathrm{~dB}$. We can observe in the first row of Tab. 2 that one obtains $I Q_{\min }=15.2 \mathrm{~dB}$ under monochromatic illumination, and $I Q_{\min }=13.5 \mathrm{~dB}$ under wide spectrum illumination, which confirms the difference of quality observed on the images.

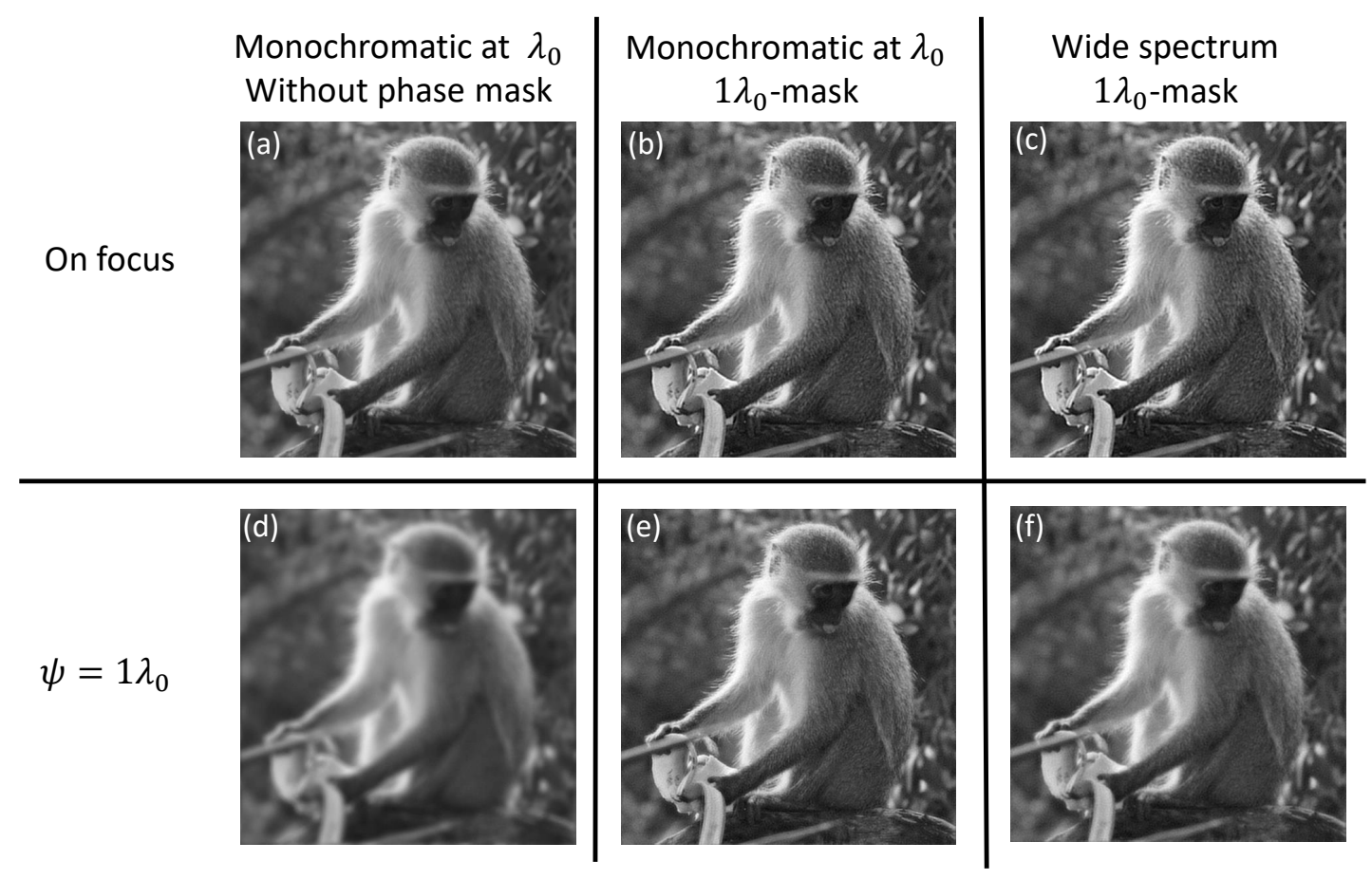

Fig 7 Simulated defocused images given by a standard optical system for (a) a focused object (d) a defocused object at $\psi=1 \lambda_{0}$. Simulated defocused images given by the entire hybrid imaging system containing the $1 \lambda_{0}$-mask (simulation of the blurred images and deconvolution) for (b) a monochromatic illumination at $\lambda_{0}$ of the object on focus (c) a wide spectrum illumination of the object on focus (e) a monochromatic illumination at $\lambda_{0}$ of the object defocused of $1 \lambda_{0}$ (f) a wide spectrum illumination of the object defocused of $1 \lambda_{0}$.

For the $2 \lambda_{0}$-mask, the improvement is also largely noticeable compared to a defocused system without phase mask (Fig. 8(d)), even if it does not recover the performance of the focused system (Fig. 8(a)). The differences between the restored images under nominal monochromatic illumination (Fig. 8(b) and (e)) and wide spectrum illumination (Fig. 8(c) and (f)) are slighter than for the $1 \lambda_{0}$-mask, but for large defocus values, the restored image is still more blurry under wide spectrum illumination (Fig. 8(f)) than under monochromatic illumination (Fig. 8(e)). This difference is confirmed by the quantitative results in the third row of Table 2: $I Q_{\min }=13.2 \mathrm{~dB}$ in the presence of monochromatic illumination at $\lambda_{0}$, whereas it is equal to $12.0 \mathrm{~dB}$ for wide spectrum illumination.

However, it can be noticed that high frequency deconvolution artifacts appear under nominal 


\begin{tabular}{|c|c|c|}
\hline Phase mask & Monochromatic illumination at $\lambda_{0}$ & Wide spectrum illumination \\
\hline $1 \lambda_{0}$-mask & $15.2 \mathrm{~dB}$ & $13.2 \mathrm{~dB}$ \\
\hline $1 \lambda_{0}$-polychromatic mask & $14.3 \mathrm{~dB}$ & $15.2 \mathrm{~dB}$ \\
\hline $2 \lambda_{0}$-mask & $13.5 \mathrm{~dB}$ & $12.0 \mathrm{~dB}$ \\
\hline $2 \lambda_{0}$-polychromatic mask & $12.4 \mathrm{~dB}$ & $13.9 \mathrm{~dB}$ \\
\hline
\end{tabular}

Table $2 I Q_{\min }$ for the phase masks optimized under monochromatic illumination assumption at $\lambda_{0}\left(1 \lambda_{0}\right.$-mask and $2 \lambda_{0}$-mask) and optimized under wide spectrum illumination assumption ( $1 \lambda_{0}$-polychromatic mask and $2 \lambda_{0^{-}}$ polychromatic mask introduced in Section 5). They are used under monochromatic illumination or wide spectrum illumination. It is assummed that the object PSD $S_{o o}(\nu)$ is proportional to $\nu^{-2.5}$ and the SNR is $34 \mathrm{~dB}$.

monochromatic illumination. They can be observed on the edges of the monkey's banana and above, at the level of its right hand in Fig. 8(c). These artefacts are a well-known drawback of DoF-enhancing phase masks with PSFs (and corresponding MTFs) that are not strictly invariant with defocus. If the averaged MTF of the average Wiener filter is too different from the actual MTF at a given value of $\psi$, deconvolution artifacts can appear for this value of $\psi$. Depending on the application, these artifacts can be really deceptive, to the point that a slightly blurry image is preferable.

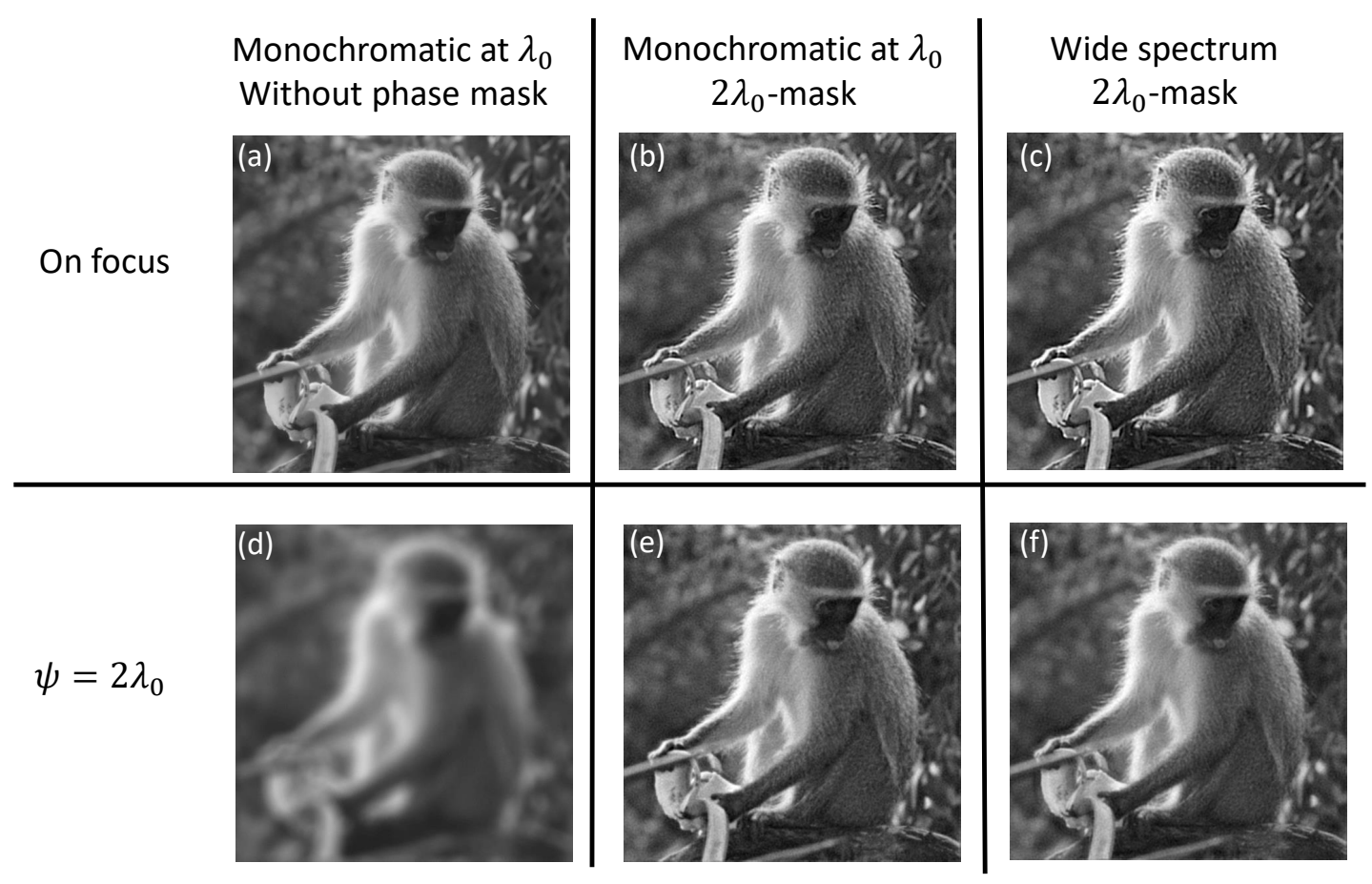

Fig 8 Simulated defocused images given by a standard optical system for (a) a focused object (d) a defocused object at $\psi=2 \lambda_{0}$. Simulated defocused images given by the entire hybrid imaging system containing the $2 \lambda_{0}$-mask (simulation of the blurred images and deconvolution) for (b) a monochromatic illumination of the object on focus (c) a wide spectrum illumination of the object on focus (e) a monochromatic illumination at $\lambda_{0}$ of the object defocused of $2 \lambda_{0}$ (f) a wide spectrum illumination of the object defocused of $2 \lambda_{0}$.

In conclusion, we have confirmed within image simulations that the masks optimized under 
monochromatic illumination are robust to wide spectrum illumination. Moreover, performance under monochromatic and wide spectrum illumination get closer as the targeted DoF range widens. The main difference is the presence of significant deconvolution artifacts when the hybrid system is used under monochromatic illumination. In contrast, under wide spectrum illumination, images are slightly blurry for some defocus values, but deconvolution artifacts are less present.

\section{Experimental validation}

In this section, we experimentally verify the conclusions drawn in the previous section from simulated images. For that purpose, we use a real imaging setup.

\subsection{Description of the optical system}

In order to investigate the behavior of co-designed binary phase masks used in a standard optical system under real lighting conditions, we have built a hybrid optical-digital setup consisting of two blocks : a co-designed phase mask and a standard lens. The lens (Edmund Optic, \#59-873) has a fixed focal length of $f_{\lambda_{0}}=50 \mathrm{~mm}$. We modify the original position of the aperture stop by placing the phase mask in a new stop a few millimeters in front of the lens (Fig. 9). The diameter of the new aperture stop is set to a somewhat low value of $4.5 \mathrm{~mm}$ in order to avoid any vignetting problem with the lens internal iris fully open. This corresponds to a F-number of 11.1 and a numerical aperture of $N A=0.045$ when the lens is focused at infinity. We have checked (thanks to a "Zemax ${ }^{\circledR}$ Black Box" of the lens provided by the manufacturer) that field aberrations are not significantly impacted and are still low when we move the stop from its original iris position to the mask clear aperture in front of the lens at the same $F / 11$ aperture.

With the F-number set at 11.1, the lens has good optical characteristics, with MTFs close to the diffraction limit over the whole field of view. It is used with a sensor of size $8.5 \mathrm{~mm} \times 7.09 \mathrm{~mm}$ and a pixel pitch of $3.45 \mu \mathrm{m}$, which corresponds to a Nyquist spatial frequency of $145 \mathrm{lp} . \mathrm{mm}^{-1}$. At $F / 11.1$, this Nyquist frequency is larger than the diffraction cutoff frequency of the optical system for the largest wavelengths of the visible spectrum but not for the medium or shortest ones. The PSF is thus well sampled for red light but a little amount of aliasing cannot be avoided under blue or green illumination.

Since the system is used under wide spectrum illumination, we have to check the axial chromatic aberration of the lens. Using the already mentioned "Zemax ${ }^{\circledR}$ black box" and the Optic Studio software package, we can obtain the lens axial chromatic focal shift. The result shows that the lens is well optimized for the visible spectrum since the focal shift diagram is folded and of low extension. Therefore, in our working conditions, the axial chromatic aberration of the lens has no significant effect and will be legitimately neglected.

\subsection{Real spectrum illumination}

In order to experimentally compare the behavior of the system under monochromatic and wide spectrum illumination, we select two different light sources whose spectra have similar properties to those used for simulations in Section 3.

The first source is a fluorescent lamp with rare-earth phosphor, giving a typical "cool white" lighting but possessing a line spectrum. In particular, it possesses two narrow peaks at $542.4 \mathrm{~nm}$ and $546.5 \mathrm{~nm}$ produced by terbium and mercury. To bring this spectrum as close as possible to a monochromatic one, we use a green filter (Edmund optics, \#46-546) centered on the wavelength 


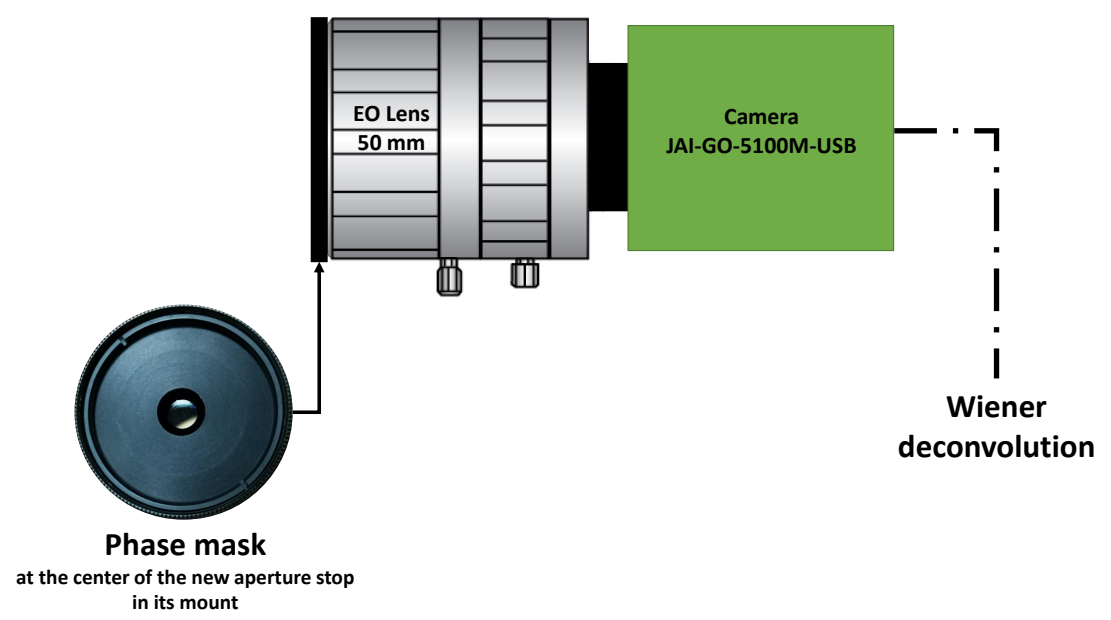

Fig 9 Scheme of the evaluated hybrid system.

$\lambda_{0}=545 \mathrm{~nm}$ and of spectral width $40 \mathrm{~nm}$ which allows to select these two peaks. We obtain in this way an effective spectrum with a high peak very close to our reference wavelength $\lambda_{0}$, that will be used to check the imaging system behavior under a quasi-monochromatic spectrum. For the second source intended to produce the wide spectrum illumination, we have chosen a halogen lamp. The effective spectra $E(\lambda)$ of these two sources, considering that the images are acquired with a CMOS silicium sensor, are represented in Fig. 4 (Fluorescent lamp with green filter and Halogen lamp).

\subsection{Experimental robustness under wide spectrum illumination}

In order to validate the robustness of binary phase masks to wide spectrum illumination, we created a scene with several objects at different defocus. It consists of sentences written on sheets of paper (of about $6 \mathrm{~cm}$ width) placed at different distances from the camera. Each sheet of paper contains four parts: a spoke target in the left side, the "name" of the imaging plane written in large font size, a sentence in medium font size and a tiny number. Each part has its interest: the spoke target allows visual evaluation of the reconstruction quality for gradually varying spatial frequencies. The large font size of the plane name enables its direct recognition. The sentence with a medium font size is a "lorem ipsum" text (consisting of random Latin words, requiring a real reading of the characters to determine whether the sentence is readable or not). The font size has been chosen to be large enough to be readable with a small amount of blur. Finally, the tiny number is only readable if the image is sharp.

Figure 10 represents an image of this scene with a conventionnal imaging system (i.e. without phase mask nor deconvolution) under quasi-monochromatic illumination (see Fig. 4 - Fluorescent lamp). The lens is focused at $50 \mathrm{~cm}$, on the plane named "Focus plane". It has a natural DoF range $\left(|\psi| \leq \frac{\lambda_{0}}{4}\right)$ within which all elements seem to be sharp. This natural DoF range is delimited by two sheets of paper on both sides of the focus plane: the first one, named "First plane - no phase mask", is placed at $48.4 \mathrm{~cm}$ and the second one, named "Last plane - no phase mask", at $51.7 \mathrm{~cm}$.

By adding the $1 \lambda_{0}$-mask in front of the optical system, we try to extend the DoF range to $45.1 \mathrm{~cm}$ and $56 \mathrm{~cm}$ after deconvolution. Therefore, we place two other sheets of paper at these 


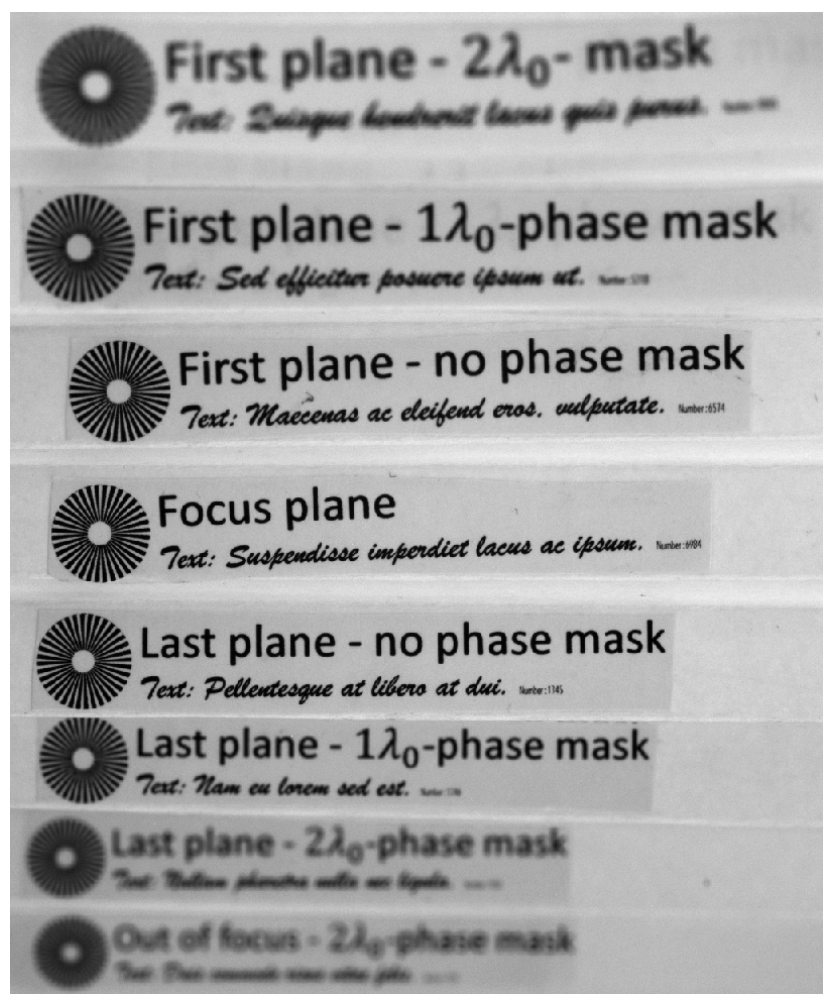

Fig 10 Scene imaged without mask and under quasi-monochromatic illumination.

distances, named "First plane - $1 \lambda_{0}$-phase mask" and "Last plane - $1 \lambda_{0}$-phase mask", to control the behavior of the hybrid system with the $1 \lambda_{0}$-mask. Similarly, by adding the $2 \lambda_{0}$-mask in front of the optical system, we try to extend the DoF until $41.1 \mathrm{~cm}$ and $63.7 \mathrm{~cm}$ after deconvolution. We place two sheets of paper at these distances, named "First plane $-2 \lambda_{0}$-phase mask" and "Last plane - $2 \lambda_{0}$-phase mask". We acquire six different images of this scene with the lens without phase mask, with the $1 \lambda_{0}$-mask and with the $2 \lambda_{0}$-mask, each for both illumination spectrums. The images acquired with a mask are restored with the average Wiener filter adapted to each mask and to the illumination spectrum with which they have been acquired. For these filters, the white noise PSD $S_{n n}$ is set to give a SNR of $28 \mathrm{~dB}$, which is closer to the real experimental conditions than the $34 \mathrm{~dB}$ SNR considered previously during mask optimization.

Figure 11(a) represents images of the whole scene under wide spectrum illumination with the $1 \lambda_{0}$-mask, and Fig. 11(b) with the $2 \lambda_{0}$-mask. We notice that, even if the phase masks are not optimized for wide spectrum illumination, they correctly reach the targeted DoF ranges. The text of the first plane of the $2 \lambda_{0}$-mask, unreadable without phase mask (Fig. 10), becomes readable with the use of the $1 \lambda_{0}$-mask and is sharp with the $2 \lambda_{0}$-mask. Even the text in the out of focus plane becomes readable with the $2 \lambda_{0}$-mask.

Let us now consider details of the scene. We observe in Fig. 12(d) that under wide spectrum illumination and without phase mask, the spoke target of the first focus plane for the $2 \lambda_{0}$-mask ("First plane $-2 \lambda_{0}$-mask") has a contrast inversion, whereas this same spoke target has only one contrast nulling with the use of the $1 \lambda_{0}$-mask (Fig. 12(e)). With the use of the $2 \lambda_{0}$-mask, the spoke target has no longer any nulling. Nevertheless, as we have seen in Section 3, the hybrid system is slightly less efficient under wide spectrum illumination than under quasi-monochromatic 
(a)

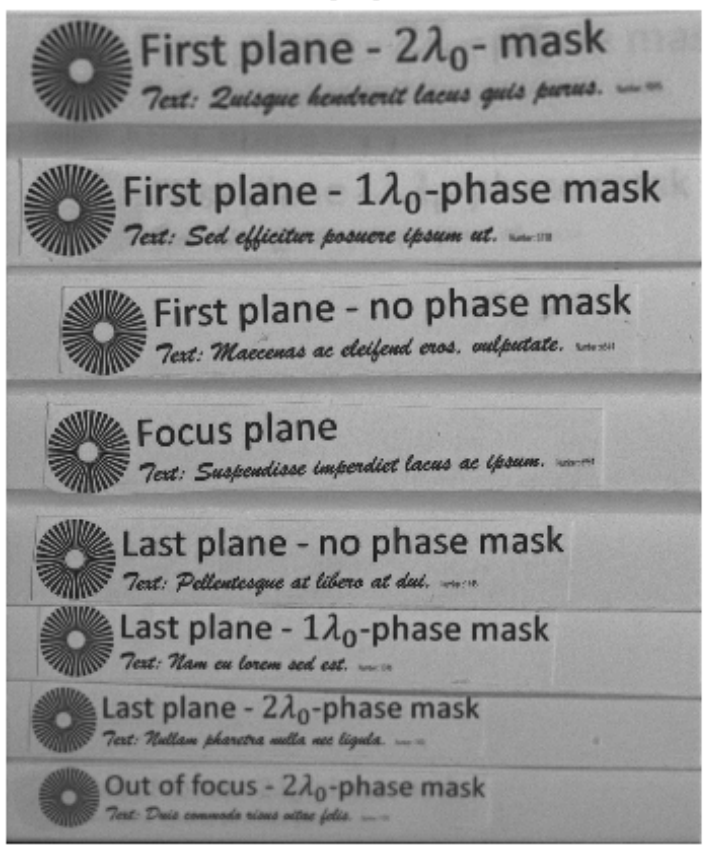

(b)

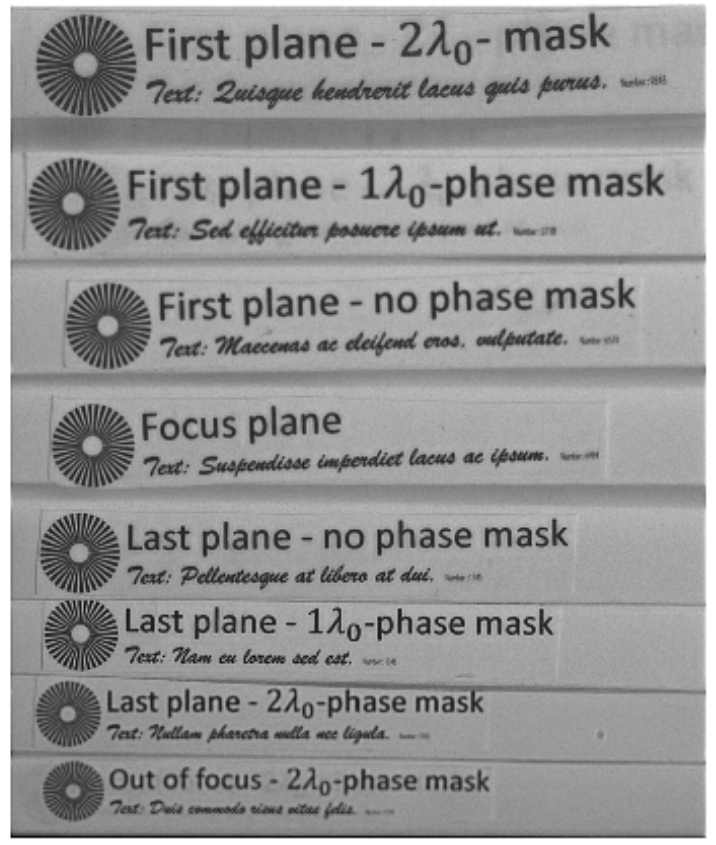

Fig 11 Image experimentally obtained under wide spectrum illumination, after deconvolution (a) with the $1 \lambda_{0}$-mask (b) with the $2 \lambda_{0}$-mask.

illumination. The imaging system used without phase mask gives similar results under quasimonochromatic spectrum (Fig. 12(a)) and (Fig. 12(d)). However, with the $1 \lambda_{0}$-mask, higher frequencies are restored under quasi-monochromatic illumination (Fig. 12(b)) than under wide spectrum illumination (Fig. 12(e)), since the center of the spoke target is blurrier in the second case. The same kind of difference is observable for the $2 \lambda_{0}$-mask: the spoke target is slightly sharper under quasi-monochromatic illumination (Fig. 12(c)) than under wide spectrum illumination (Fig. 12(f)). The lower quality under wide spectrum illumination at the edge of the DoF range was already observed with the $2 \lambda_{0}$-mask in the simulated images (Fig. 8(c) and Fig. 8(d)).

Figure 13 represents another interesting detail of the scene: it consists of the tiny number of the first sharp plane for the $1 \lambda_{0}$-mask ("First plane - $1 \lambda_{0}$-phase mask"). It is not readable without phase mask, regardless of illumination (Fig. 13(a) and Fig. 13(b)). With the $1 \lambda_{0}$-mask, the number "5318" becomes readable under quasi-monochromatic illumination (Fig. 13(c)) and under wide spectrum illumination (Fig. 13(d)). Reconstruction is slightly better under quasi-monochromatic illumination. Therefore, the $1 \lambda_{0}$-mask is robust to wide spectrum illumination but the image quality is reduced at the edge of the optimized DoF range.

Therefore, the two phase masks are robusts to wide spectrum illumination but slightly less efficient than under monochromatic illumination at $\lambda_{0}$. However, we have previously noticed that the deconvolution process produced visible oscillations on simulated images for some defocus positions under monochromatic illumination. These oscillations are experimentally clearly visible. In Fig. 14(b), the text at the focus plane obtained with the $2 \lambda_{0}$-mask under quasi-monochromatic illumination has a white halo all around, although the text is as sharp as when there is no phase mask (Fig. 14(a)). They are not present under wide spectrum illumination (Fig. 14(c)), the counterpart 


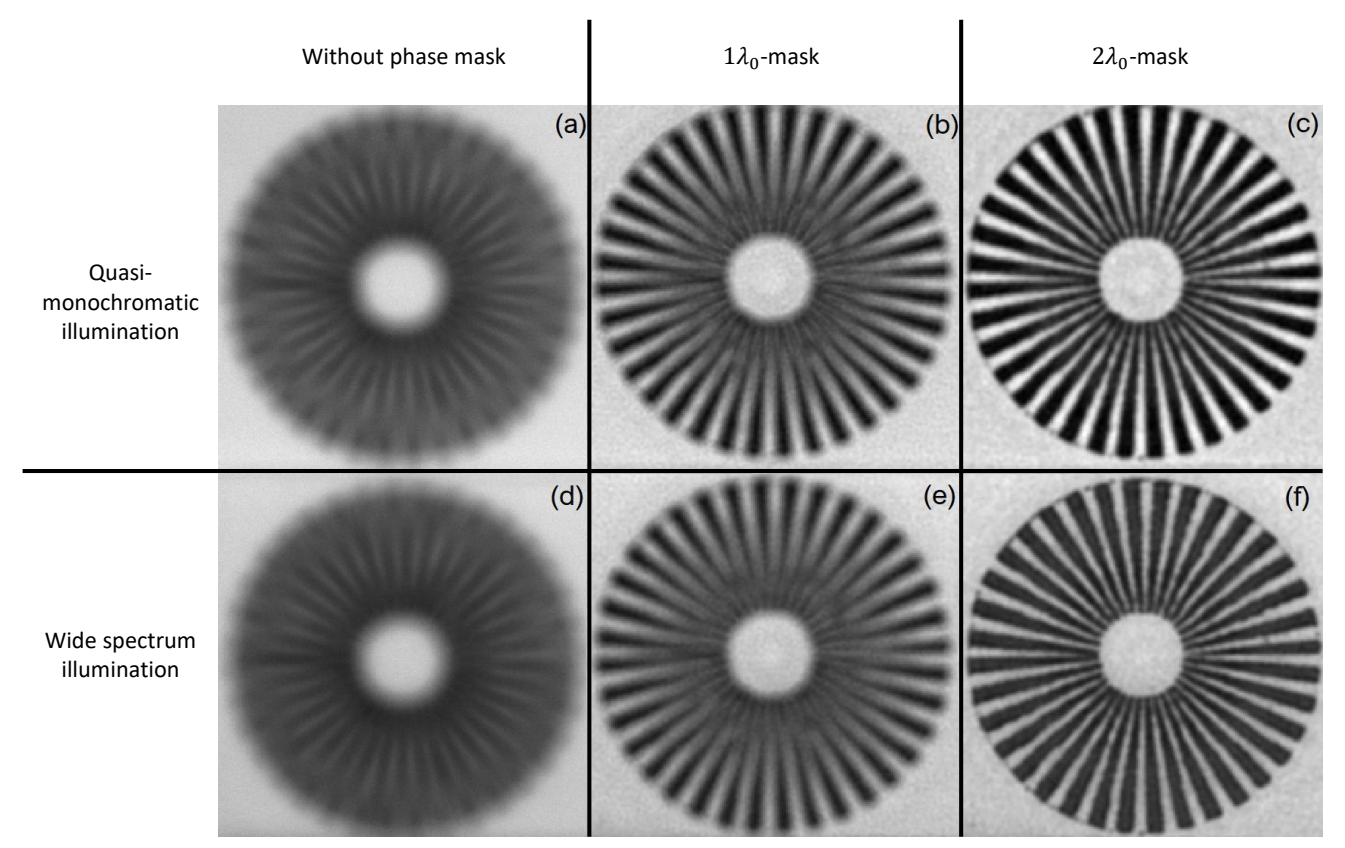

Fig 12 Zoom on the spoke target of the "First plane- $2 \lambda_{0}$-mask" of Fig. 11, under quasi-monochromatic illumination (first row) and wide spectrum illumination (second row) in different configurations. (a \& d) Optical system without phase mask. (b \& e) Hybrid system with the $1 \lambda_{0}$-mask and deconvolution. (c \& f) Hybrid system with the $2 \lambda_{0}$-mask and deconvolution.

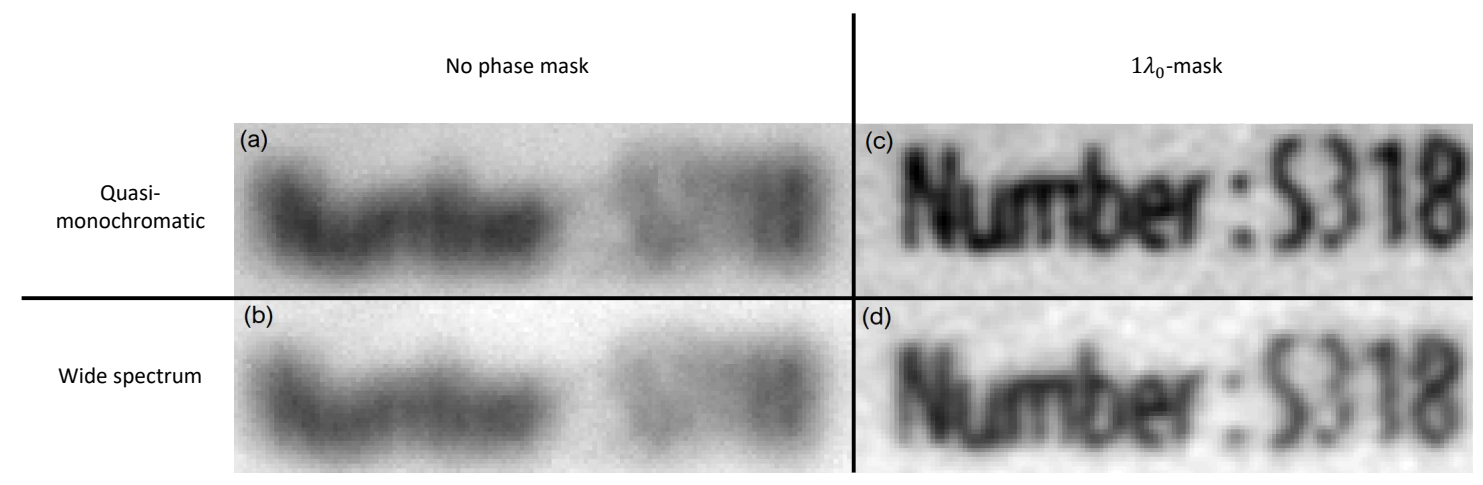

Fig 13 Zoom on the number of the "First plane - $1 \lambda_{0}$-phase mask", for quasi-monochromatic illumination (firt column) and wide spectrum illumination (second column) with different imaging systems. (a \& b) Without phase mask (c \& d) With the $1 \lambda_{0}$-mask and deconvolution.

being that the text is less sharp.

Thus, we have experimentally demonstrated the robustness of the binary phase masks to wide spectrum illumination. The main conclusion is the same as that drawn for the simulated images in Section 3.2: in the presence of wide spectrum illumination, restored images are slightly more blurry at the edge of the targeted DoF range, but deconvolution artifacts are significantly reduced. 


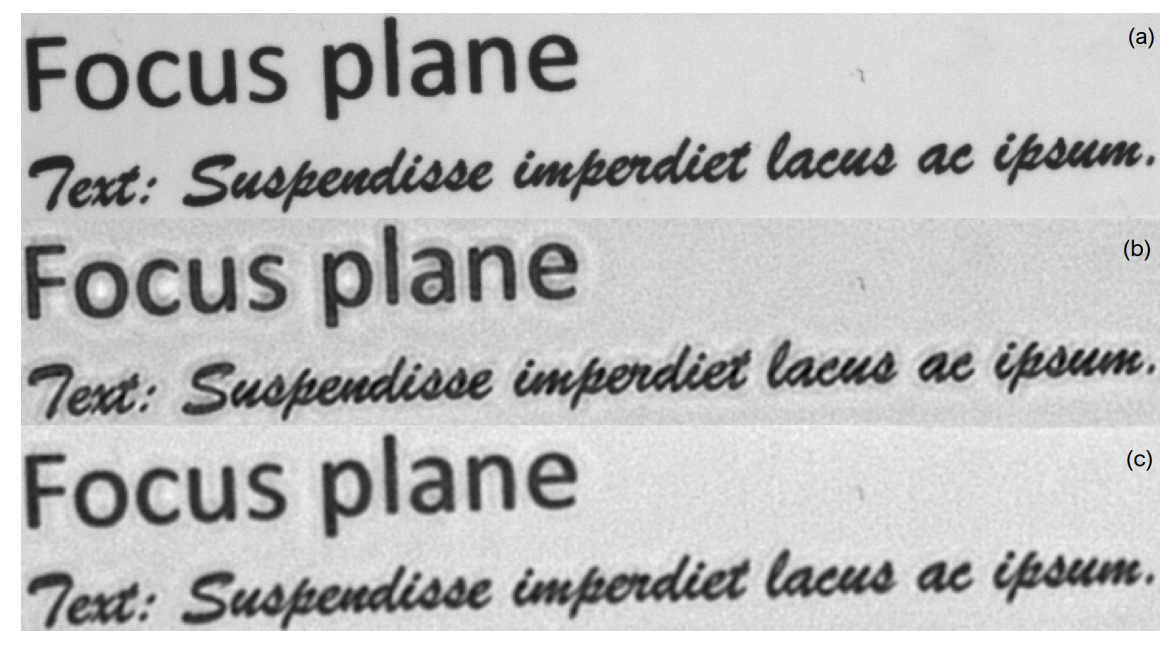

Fig 14 Zoom on the text of the focus plane, with different imaging systems. (a) Optical system without phase mask under quasi-monochromatic illumination (b) Hybrid system with the $2 \lambda_{0}$-mask under quasi-monochromatic illumination (c) Hybrid system with the $2 \lambda_{0}$-mask under wide spectrum illumination.

\section{Optimizing masks assuming a wide spectrum illumination}

We have shown that the phase masks optimized assuming monochromatic illumination are robust under wide spectrum illumination. However, one can wonder whether taking into account wide spectrum illumination at the optimization stage would yield significantly better performance. The purpose of the present section is to investigate this issue. To perform optimization under wide spectrum, in addition to the radii of the rings, another parameter has to be optimized : the common step height of the binary phase mask rings. Indeed, under monochromatic assumption, choosing a step height corresponding to a phase shift of $\pi$ radians is natural since it is the only one that allows a symmetrical behavior of the phase mask with respect to the defocus parameter, making it possible to double the reachable DoF. Under wide spectrum assumption, the choice is not so obvious, and we have noticed that the step height corresponding to a phase shift of $\pi$ radians at the barycentric wavelength of the spectrum does not lead to maximal image quality. In consequence, we considered the step height as a free parameter in mask optimization. Using this approach, we optimized two binary phase masks for the two targeted DoF ranges $\left[-1 \lambda_{0}, 1 \lambda_{0}\right]$ and $\left[-2 \lambda_{0}, 2 \lambda_{0}\right]$, with still $\lambda_{0}=545 \mathrm{~nm}$ and with the sun spectrum illumination assumption (Fig. 4). We will denote them " $1 \lambda_{0}$-polychromatic mask" and " $2 \lambda_{0}$-polychromatic mask" in the following.

Let us first consider the $1 \lambda_{0}$-polychromatic mask. Its optimal step height and normalized ring radii appear in the second row of Tab. 1. It is noticed that the optimal step height is significantly different from that of the $1 \lambda_{0}$-mask ( $722 \mathrm{~nm}$ instead of $522 \mathrm{~nm}$ ). Moreover, the rings are positioned at different places. The value of $I Q_{\min }$ computed for an image with generic PSD appears in the second column of Tab. 2. This value is significantly larger than the one obtained with the $1 \lambda_{0}$-mask optimized under the hypothesis of monochromatic light (15.2 dB instead of $\left.13.2 \mathrm{~dB}\right)$, showing that there is a significant benefit in taking into account the actual illumination spectrum in mask optimization. In order to further illustrate these results, we have simulated the restored images obtained in the considered configurations using the scene "Monkey" (see the two first rows of Fig. 15). We can see in Figures 15(a) and 15(d) that when the mask has been optimized for the illumination used in image simulation (monochromatic illumination for $1 \lambda_{0}$-mask and wide 
spectrum illumination for $1 \lambda_{0}$-polychromatic mask), the image quality is satisfying, and much better that in Fig. 15(b), where the image is too blurry, and in Fig. 15(c), where deconvolution artifacts are visible.

These conclusions are confirmed and amplified when $\psi_{\max }=2 \lambda_{0}$. In the fourth row of Tab. 1 , it is seen that the $2 \lambda_{0}$-polychromatic mask has only five rings instead of six for the $2 \lambda_{0}$-mask. Moreover, the optimal step height is significantly different (694 nm instead of $522 \mathrm{~nm}$ ). In the fourth column of Tab. 2, it is seen that in the presence of wide spectrum illumination, the value of $I Q_{\min }$ obtained with the $2 \lambda_{0}$-polychromatic mask is significantly larger than the one obtained with the $2 \lambda_{0}$-mask $(13.9 \mathrm{~dB}$ instead of $12.0 \mathrm{~dB})$. Interestingly, this value is even larger than the $I Q_{\min }$ obtained with the $2 \lambda_{0}$-mask in the presence of nominal monochromatic illumination (13.5 $\left.\mathrm{dB}\right)$. This fact is corroborated by the image simulations in Fig. 15, since the $I Q_{\min }$ of Fig. 15(h) is $0.6 \mathrm{~dB}$ larger that of Fig. 15(e). This result is unexpected, since the binary phase mask may be thought to have better performance when optimized for a single wavelength, whereas optimization in the presence of wide spectrum is the result of a compromise between different wavelength contributions. However, it has been seen in the previous section that in the presence of wide spectrum illumination, the through-focus variations of the MTF are smoother due to an averaging effect of different wavelengths (see Figures 3 and 5). We have shown that this result implies that restored images are blurrier but have less deconvolution artifacts than under monochromatic illumination (see Fig. 14). When the mask is optimized for wide spectrum instead of monochromatic illumination, the "blurrying effect" is reduced since the mask is more adapted to the actual illumination, and the advantage of having smooth MTF variations remains. In consequence, the image in Fig. 15(h) is reasonably sharp and devoid of artifacts, whereas Fig. 15(e) is plagued with strong deconvolution artifacts, which explains the large difference in terms of $I Q_{\min }$ values.

In conclusion, taking into account wide spectrum illumination in mask optimization is important since it makes it possible to obtain better performance in terms of $\mathrm{IQ}_{\text {min }}$, especially when the targeted DoF range is wide. Moreover, DoF extension is easier to reach in the presence of wide spectrum illumination than of monochromatic illumination, since it reduces the deconvolution artifacts that are due to the sharp through-focus MTF variations observed in monochromatic imaging.

\section{Conclusion}

We have studied optimization and performance of DoF enhancing phase masks in the presence of monochromatic or wide spectrum illumination. Through theoretical and experimental study, we have shown that phase masks optimized under monochromatic illumination assumption are somewhat robust to their use under wide spectrum illumination. They provide, after digital image restoration, images that are slightly less sharp than under monochromatic illumination but are less affected by deconvolution artifacts thanks to spectral averaging. When the masks are optimized under wide spectrum assumption, their performance in the presence of this type of illumination becomes higher than that of monochromatically optimized masks under nominal monochromatic illumination, especially when the targeted DoF range is large. This interesting effect comes from the fact that deconvolution artifacts are significantly reduced by wide spectrum illumination. These results are important since they show that it is useful to take into account the spectral width of illumination together with the scene characteristics and the targeted DoF range for efficiently codesign DoF enhancing imaging systems. 


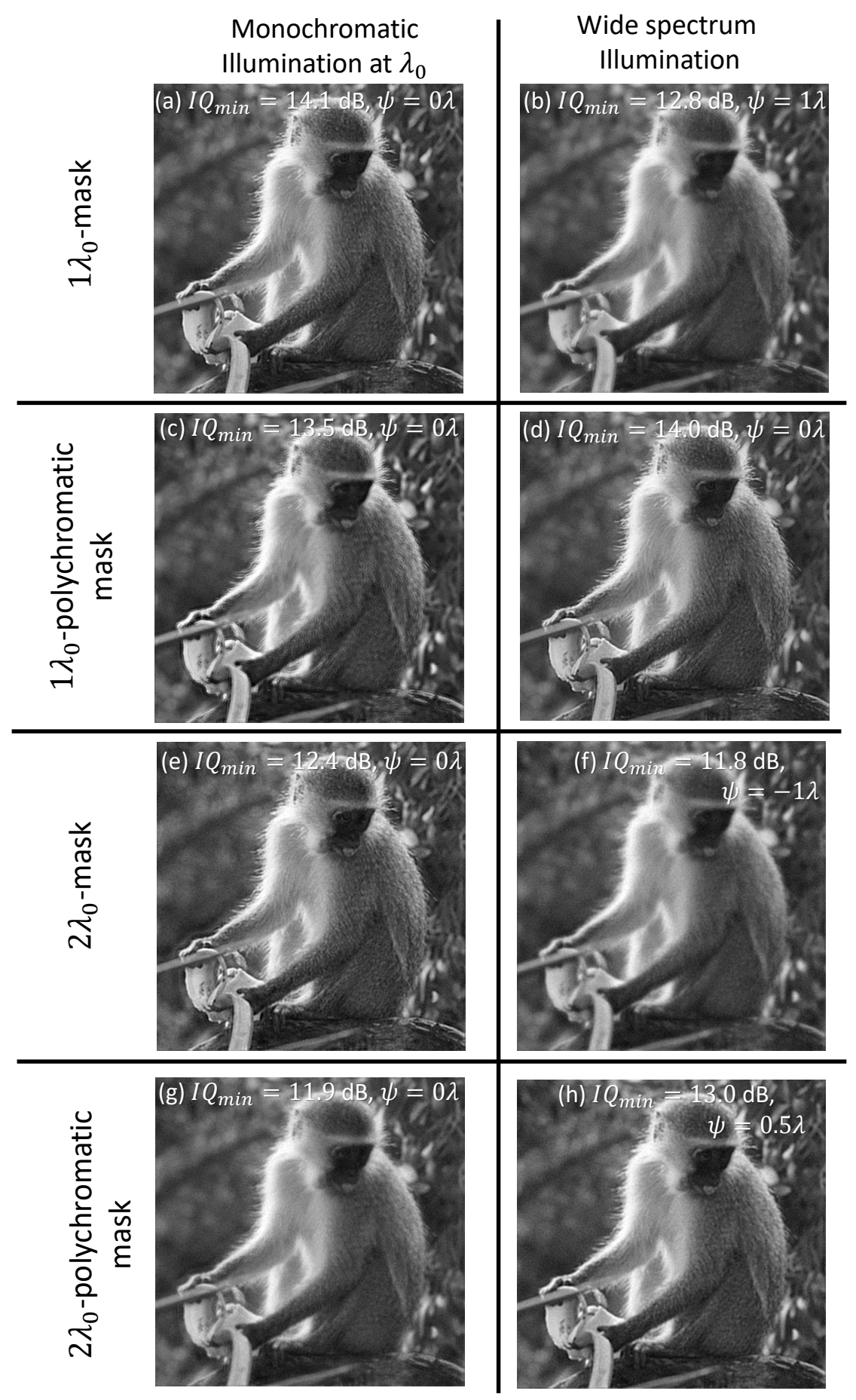

Fig 15 Restored images for different illuminations (column) and different masks (rows). In each case, the image corresponding to the worse image quality over the DoF range is represented. The value of defocus parameter $\psi$ for which is image is obtained, and the corresponding value of $I Q_{\min }$ are given on each image.

This work has many perspectives. The conclusions obtained in this study should be confirmed in cases when the F-number of the lens is smaller and the chromatic aberration of the underlying 
lens without phase mask larger, in order to investigate the interplay between field aberrations, chromatic aberration and DoF extension. The present approach of DoF extension could also be extended to RGB color imaging.

\section{Acknowledgments}

The authors wish to gratefully thank Anne-Lise Coutrot for the manufacturing of phase masks, with the help of the microfabrication platform at Thales Research and Technology Palaiseau and especially Julie Cholet and Etienne Eustache. The authors also thank Jean-René Rullier for his help with the holding of phase masks in front of the lens, and Olivier Lévêque for the monkey picture. The work reported in this study is supported in part by the Agence de l'Innovation de Défense (AID) that provides half of a PhD fellowship to Alice Fontbonne. Authors have no conflict of interest related to the work leading to the results presented in this manuscript.

\section{References}

1 E. R. Dowski and W. T. Cathey, "Extended depth of field through wave-front coding," Appl. Opt. 34, 1859-1866 (1995).

2 W. T. Cathey and E. R. Dowski, "New paradigm for imaging systems," Applied optics 41(29), 6080-6092 (2002).

3 A. Castro, Y. Frauel, and B. Javidi, "Integral imaging with large depth of field using an asymmetric phase mask," Optics Express 15, 10266 (2007).

4 F. Zhou, R. Ye, G. Li, et al., "Optimized circularly symmetric phase mask to extend the depth of focus," Journal of the Optical Society of America A 26, 1889 (2009).

5 X. Mo, "Optimized annular phase masks to extend depth of field," Optics Letters 37, 1808 (2012).

6 N. Caron and Y. Sheng, "Polynomial phase masks for extending the depth of field of a microscope," Appl. Opt. 47, E39-E43 (2008).

7 V. Katkovnik, N. Hogasten, and K. Egiazarian, "A novel binary and multilevel phase masks for enhanced depth-of-focus infrared imaging," 386-390 (2018).

8 E. Ben-Eliezer, N. Konforti, B. Milgrom, et al., "An optimal binary amplitude-phase mask for hybrid imaging systems that exhibit high resolution and extended depth of field," Optics express 16(25), 20540-20561 (2008).

9 T. Zhao, A. Liu, Q. Liu, et al., "Axial intensity distribution analysis for a depth-of-fieldextended optical system using a low-frequency binary phase mask," Applied optics 53(17), 3782-3786 (2014).

10 Z. Zalevsky, A. Shemer, A. Zlotnik, et al., "All-optical axial super resolving imaging using a low-frequency binary-phase mask," Opt. Express 14, 2631-2643 (2006).

11 S. Ryu and C. Joo, "Design of binary phase filters for depth-of-focus extension via binarization of axisymmetric aberrations," Opt. Express 25, 30312-30326 (2017).

12 F. Diaz, F. Goudail, B. Loiseaux, et al., "Increase in depth of field taking into account deconvolution by optimization of pupil mask," Optics letters 34(19), 2970-2972 (2009).

13 D. Robinson and D. G. Stork, "Joint design of lens systems and digital image processing," in International Optical Design, International Optical Design, WB4, Optical Society of America (2006). 
14 S. Elmalem, R. Giryes, and E. Marom, "Learned phase coded aperture for the benefit of depth of field extension,” Opt. Express 26, 15316-15331 (2018).

15 R. Falcón, F. Goudail, C. Kulcsár, et al., "Performance limits of binary annular phase masks codesigned for depth-of-field extension," Optical Engineering 56(6), 065104 (2017).

16 A. Fontbonne, H. Sauer, C. Kulcsár, et al., "Experimental validation of hybrid optical-digital imaging system for extended depth-of-field based on co-optimized binary phase masks," $O p$ tical Engineering 58, 1 (2019).

17 H. Haim, A. Bronstein, and E. Marom, "Computational multi-focus imaging combining sparse model with color dependent phase mask," Optics Express 23, 24547 (2015).

18 B. Milgrom, N. Konforti, M. A. Golub, et al., "Pupil coding masks for imaging polychromatic scenes with high resolution and extended depth of field," Optics express 18(15), 15569-15584 (2010).

19 B. Milgrom, N. Konforti, M. A. Golub, et al., "Novel approach for extending the depth of field of barcode decoders by using rgb channels of information," Opt. Express 18, 1702717039 (2010).

20 S. Elmalem, N. Konforti, and E. Marom, "Polychromatic imaging with extended depth of field using phase masks exhibiting constant phase over broad wavelength band," Applied optics 52(36), 8634-8643 (2013).

21 B. Milgrom, R. Avrahamy, T. David, et al., "Extended depth-of-field imaging employing integrated binary phase pupil mask and principal component analysis image fusion," Opt. Express 28, 23862-23873 (2020).

22 S. Elmalem, R. Giryes, and E. Marom, "Motion deblurring using spatiotemporal phase aperture coding," (2020).

23 P. Trouvé, F. Champagnat, G. L. Besnerais, et al., "Passive depth estimation using chromatic aberration and a depth from defocus approach," Applied Optics 52, 7152 (2013).

24 J. Garcia, J. Sanchez, X. Orriols, et al., "Chromatic aberration and depth extraction," in Proceedings 15th International Conference on Pattern Recognition. ICPR-2000, IEEE Comput. Soc.

25 F. Diaz, M.-S. L. Lee, X. Rejeaunier, et al., "Real-time increase in depth of field of an uncooled thermal camera using several phase-mask technologies," Optics letters 36(3), 418-420 (2011).

26 M.-A. Burcklen, F. Diaz, F. Lepretre, et al., "Experimental Demonstration of Extended Depth-of-Field F/1.2 Visible High Definition Camera with Jointly Optimized Phase Mask and Real-Time Digital Processing," Journal of the European Optical Society 10, 150461150466 (2015).

27 J. W. Goodman, Introduction to Fourier Optics, 3th edition, Roberts and Company Publishers (2005).

28 D. L. Ruderman, “Origins of scaling in natural images," Vision Research 37(23), 3385 - 3398 (1997).

29 A. van der Schaaf and J. van Hateren, "Modelling the power spectra of natural images: Statistics and information," Vision Research 36(17), 2759 - 2770 (1996). 
Alice Fontbonne received her MS degree in optics from Institut dOptique Graduate School. She is now a PhD fellow at Institut dOptique Graduate School (Palaiseau, France) and works on joint design of optics and image processing as a new approach to high level optical design.

Hervé Sauer is a former student of the École Normale Supérieure de Fontenay/St-Cloud. He got the Agregation degree in physics in 1985, and a MSc and $\mathrm{PhD}$ in physics from Université Paris Sud-XI (Orsay) in 1985 and 1990 respectively. He is an associate professor at Institut d'Optique Graduate School (Palaiseau) since 1990. His main research topics deal with simulations of optical systems and optical system design.

François Goudail graduated from the École Supérieure d'Optique, (Orsay), in 1992 and obtained his $\mathrm{PhD}$ in 1997 from the University of Aix-Marseille III. He has been associate professor at Fresnel Institute, (Marseille), until 2005. He is now professor at the Institut dOptique Graduate School (Palaiseau). His research topics include information extraction in images from different types of passive and active sensors (hyperspectral, SAR, polarimetric), wavefront engineering and joint design of optical systems and image processing algorithms. 